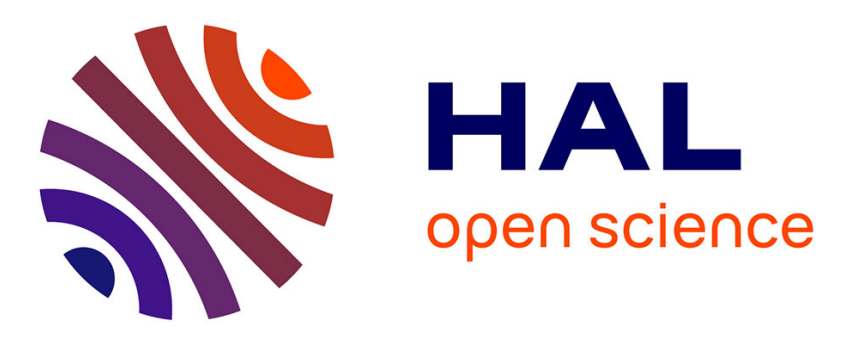

\title{
Locally implicit discontinuous Galerkin time domain method for electromagnetic wave propagation in dispersive media applied to numerical dosimetry in biological tissues
}

\author{
Stéphane Descombes, Stéphane Lanteri, Ludovic Moya
}

\section{To cite this version:}

Stéphane Descombes, Stéphane Lanteri, Ludovic Moya. Locally implicit discontinuous Galerkin time domain method for electromagnetic wave propagation in dispersive media applied to numerical dosimetry in biological tissues. SIAM Journal on Scientific Computing, 2016, 38 (5), pp.A2611-A2633. 10.1137/15M1010282. hal-01133694v2

\section{HAL Id: hal-01133694 \\ https://hal.inria.fr/hal-01133694v2}

Submitted on 23 Sep 2016

HAL is a multi-disciplinary open access archive for the deposit and dissemination of scientific research documents, whether they are published or not. The documents may come from teaching and research institutions in France or abroad, or from public or private research centers.
L'archive ouverte pluridisciplinaire HAL, est destinée au dépôt et à la diffusion de documents scientifiques de niveau recherche, publiés ou non, émanant des établissements d'enseignement et de recherche français ou étrangers, des laboratoires publics ou privés. 


\title{
LOCALLY IMPLICIT DISCONTINUOUS GALERKIN TIME DOMAIN METHOD FOR ELECTROMAGNETIC WAVE PROPAGATION IN DISPERSIVE MEDIA APPLIED TO NUMERICAL DOSIMETRY IN BIOLOGICAL TISSUES
}

\author{
STÉPHANE DESCOMBES*†, STÉPHANE LANTERI ${ }^{\dagger}$, AND LUDOVIC MOYA ${ }^{\dagger}$
}

\begin{abstract}
We are concerned here with the numerical simulation of electromagnetic wave propagation in biological media. Because of their water content, these media are dispersive i.e. their electromagnetic material characteristics depend of the frequency. In the time-domain, this translates in a time dependency of these parameters that can be taken into account through an additional (auxiliary) differential equation for, e.g, the electric polarization, which is coupled to the system of Maxwell's equations. From the application point of view, the problems at hand most often involve irregularly shaped structures corresponding to biological tissues. Modeling realistically the interfaces between tissues is particularly important if one is interested in evaluating accurately the impact of field discontinuities at these interfaces. In this paper, we propose and study a locally implicit discontinuous Galerkin time-domain method formulated on an unstructured tetrahedral mesh for solving the resulting system of differential equations in the case of Debye-type media. Three-dimensional numerical simulations are presented concerning the exposure of head tissues to a localized source radiation.
\end{abstract}

Key words. time-domain Maxwell's equations, dispersive media, Debye, discontinuous Galerkin method, locally implicit scheme.

AMS subject classifications. 35Q61, 65L20

1. Introduction. This article is concerned with the numerical simulation of electromagnetic wave propagation in dispersive media. These are materials in which either or both of the electromagnetic material parameters $\varepsilon$ and $\mu$ are functions of frequency. Note that the conductivity $\sigma$ may also be a function of frequency, but its effect can be incorporated in the complex permittivity. In reality, all materials have frequency-dependent $\varepsilon$ and $\mu$, but many materials can be approximated as frequency-independent over a frequency band of interest, simplifying their analysis and simulation. Here, we will focus on the much more common case of frequencydependent permittivity. A lot of practical electromagnetic wave propagation problems involve such propagation media, such as those involving the interaction of an electromagnetic wave with biological tissues. The numerical modeling of the propagation of electromagnetic waves through human tissues is at the heart of many biomedical applications such as the microwave imaging of cancer tumors or the treatment of the latter by hyperthermia. For example, microwave imaging for breast cancer detection is expected to be safe for the patient and has the potential to detect very small cancerous tumors in the breast [11, 26, 37]. Beside, the definition of microwavebased hyperthermia as an immunotherapy strategy for cancer can also be cited [5, 23]. The electroporation technique can also be an application, which consists of applying nanopulses to the tissues, enabling only intracellular membranes to be affected, and then opening the route to therapeutic strategies such as electrochemotherapy or gene transfer [33, 44, 39, 40, 42]. Because for all these biomedical applications experimental modeling is almost impossible, computer simulation is the approach of choice

\footnotetext{
*Univ. Nice Sophia Antipolis, CNRS, LJAD, UMR 7351, 06100 Nice, France, (stephane.descombes@unice.fr, +33 4920762 83).

${ }^{\dagger}$ INRIA Sophia Antipolis-Mediterranée Research Center, Nachos project-team, 06902 Sophia Antipolis Cedex, France (stephane.lanteri@inria.fr, +33 4923877 34, ludovic.moya@inria.fr, + 334923876 12).
} 
for understanding the underlying physical phenomena. In order to do so, one needs accurate and efficient numerical modeling techniques, able to deal with the complex issues characterizing the associated propagation problems.

Numerical simulation of wave propagation in dispersive media started in early 1990's in the framework of Finite Difference Time Domain (FDTD) methods, for details and references see e.g. [20] or [41]. Finite Element Time Domain (FETD) methods were not explored until 2001 [21] and Discontinuous Galerkin Time Domain (DGTD) methods for solving Maxwell's equations in dispersive media have been considered more recently. DGTD methods share almost all the advantages of FETD methods (large spectrum of applications, complex geometries, etc) and FVTD methods (ability to capture discontinuous solutions). The DGTD method has other nice properties which explain the renewed interest it gains in various domains in scientific computing as witnessed by books or special issues of journals dedicated to this method $[2,4,6,15]$. Concerning Maxwell's equations in the non-dispersive case, DGTD discretizations have been introduced in [3] and [16], Concerning the dispersive case, in [30, 29], a DGTD method, which uses piecewise high-order polynomials for spatial discretization and a Runge-Kutta method for time integration, is derived for linear dispersive media of Debye type, the treatment of the dispersive character relying on an Auxiliary Differential Equation (ADE) approach. Numerical results for two-dimensional problems are given and no proof of convergence is provided. In $[18,19]$, a priori error estimates are proved for the second-order formulation of Maxwell's equations coupled to dispersive models discretized by an interior penalty DGTD formulation. Some two-dimensional numerical tests are included for supporting the analysis. In [45], different dispersive media are treated, considering a locally divergence-free DG method. The scheme is written and studied in its semi-discretized version, while the fully discrete scheme is described but not analyzed. Finally, in [27], which deals with the Debye model, the same DG method than the one considered in the present article is used with a second-order leap-frog scheme for time integration. Stability estimates are derived through energy conservation and convergence is proved for both the semi-discrete and the fully discrete scheme. A two-dimensional artificial numerical problem is presented to validate the theoretical findings. All these studies rely on fully explicit or fully implicit time schemes. Here we propose and analyze a DG method coupled with an efficient time integration method for dealing with grid induced stiffness when using non-uniform (locally refined) meshes and use these methods to study the interaction of electromagnetic waves with biological tissues. Stability and convergence analysis of the resulting scheme are studied, and three-dimensional problems are presented.This scheme belongs to the class of implicit-explicit (IMEX) scheme providing a single time step for stability, independent of the fine grid. Other methods also exist based on explicit local time-stepping techniques (see $[9,38,13]$ and [14] specially for Maxwell's equations), which consist to use smaller time steps, given by a local stability criterion, precisely where the smallest elements are located to overcome the bottleneck caused by local mesh refinement in explicit time integrators.

This paper is organized as follows. Section 2 presents the formulation of Maxwell's equations for Debye dispersive media. The Debye model is most often used to model electromagnetic wave interactions with water-based substances, such as biological materials. In particular, biological tissues are well represented by multi-pole Debye models. The dispersive character will be taken into account via an ADE which relates the electric polarization to the electric field. In Section 3 Maxwell's equations in dispersive media are discretized according to a DG formulation and we adapt the 
locally implicit time integration method from [43] with the additional ADE, The stability of the resulting locally implicit DGTD method is analyzed via an energy approach that provides a rigorous stability criterion. We also state a convergence result to prove that the locally implicit DGTD method for Maxwell's equations in dispersive media is second-order accurate. Finally, in section 4, we present some numerical results for three-dimensional problems.

2. The continous problem : Maxwell's equations in dispersive media. In this section we recall the initial and boundary value problem used to model the propagation of an electromagnetic wave in a dispersive medium. The physical behaviour of the medium is assumed to be described by the Debye model modeled by a combination of the first order Maxwell's equations and an ADE representation.

First, we recall Maxwell's equations which govern the electric field $\vec{E}$ and the magnetic field $\vec{H}$ in matter. Let $\Omega$ be a bounded smooth convex domain, let $T>0$, Maxwell's equations in $[0, T]$ are given by

$$
\left\{\begin{array}{l}
\frac{\partial \vec{B}}{\partial t}=-\operatorname{curl}(\vec{E}) \\
\frac{\partial \vec{D}}{\partial t}=\operatorname{curl}(\vec{H})-\vec{J}^{c}-\vec{J}^{s} \\
\operatorname{div}(\vec{B})=0 \\
\operatorname{div}(\vec{D})=0
\end{array}\right.
$$

where $\vec{B}$ and $\vec{D}$ are the magnetic and electric flux densities, $\vec{J}^{c}$ represents the conduction current density and $\vec{J}^{s}$ a given source current density. Introducing $\sigma$, the conductivity, Ohm's law governs the definition of the conduction current density, i.e. $\vec{J}^{c}=\sigma \vec{E}$. Moreover, we will assume that $\vec{J}^{s}=0$. Finally we will consider dielectric media for which magnetic effects are negligible. Introducing $\mu_{0}$ the magnetic permittivity in vacuum, this yields the relation $\vec{B}=\mu_{0} \vec{H}$. For linear dispersive materials, the constitutive relationship between the electric flux density and electric field phasors, valid at each frequency $\omega$ and at each point in space $\vec{x}$, can be expressed in terms of $\varepsilon_{0}$, the electric permittivity in vacuum, and $\varepsilon_{r}$ the complex relative permittivity, by the following relation

$$
\widehat{\vec{D}}(\vec{x}, \omega)=\varepsilon_{0} \varepsilon_{r}(\vec{x}, \omega) \widehat{\vec{E}}(\vec{x}, \omega)
$$

where $\widehat{\vec{D}}$ and $\widehat{\vec{E}}$ denote the Fourier transform of $\vec{D}$ and $\vec{E}$. Introducing $\widehat{\vec{P}}$ the electric polarization phasor, $\widehat{\chi}_{e}$ the frequency-domain electric susceptibility of the material and $\varepsilon_{\infty}$ the infinite frequency relative permittivity, equation (2.2) is often written as

$$
\begin{aligned}
\widehat{\vec{D}}(\vec{x}, \omega) & =\varepsilon_{0} \varepsilon_{\infty}(\vec{x}) \widehat{\vec{E}}(\vec{x}, \omega)+\widehat{\vec{P}}(\vec{x}, \omega), \\
& =\varepsilon_{0} \varepsilon_{\infty}(\vec{x}) \widehat{\vec{E}}(\vec{x}, \omega)+\varepsilon_{0} \widehat{\chi}_{e}(\vec{x}, \omega) \widehat{\vec{E}}(\vec{x}, \omega),
\end{aligned}
$$

The use of a frequency-dependent electric permittivity is linked to the fact that the electric flux density $\vec{D}$ in a dielectric resulting from an applied alternating electric field $\vec{E}$ has a different phase with respect to $\vec{E}$. This behavior is due to the inertia of the polarization $\vec{P}$ which, when frequency becomes high enough, cannot follow the rapid variations of the field, giving rise to a relaxation (i.e., a momentary delay or 
lag) of the permittivity. Due to the product of two frequency-dependent quantities in (2.3), the time-domain equivalent is given by the following convolution integral

$$
\begin{aligned}
\vec{D}(\vec{x}, t) & =\varepsilon_{0} \varepsilon_{\infty}(\vec{x}) \vec{E}(\vec{x}, t)+\vec{P}(\vec{x}, t) \\
& =\varepsilon_{0} \varepsilon_{\infty}(\vec{x}) \vec{E}(\vec{x}, t)+\varepsilon_{0} \int_{0}^{t} \vec{E}(\vec{x}, t-s) \chi_{e}(\vec{x}, s) d s .
\end{aligned}
$$

In order to avoid the direct numerical treatment of the convolution integral in (2.4), approaches based on a recursive calculation of the convolution integral (Recursive Convolution Method - RCM) on one hand, and on the integration of an auxiliary differential equation (ADE) on the other hand, have been developed for implementing (2.4), see e.g. [32, 31, 25] and [24, 22], respectively. In this article we assume that the medium is a single-pole Debye type dispersive medium and concentrate on the ADE technique. Debye media are characterized by a complex valued, frequencydomain susceptibility function $\widehat{\chi}_{e}(\omega)$ that has one or more real poles at separate frequencies. For a single-pole Debye medium, using the notation $j=\sqrt{-1}$, we have

$$
\widehat{\chi}_{e}(\omega)=\frac{\varepsilon_{s}-\varepsilon_{\infty}}{1+j \omega \tau}=\frac{\Delta \varepsilon}{1+j \omega \tau},
$$

with $\varepsilon_{s}$, called the static relative permittivity, the permittivity at zero frequency $\left(\varepsilon_{s}>\varepsilon_{\infty}\right)$ and $\tau$ the Debye relaxation time constant, characteristic of the material. With this particular functional form of the susceptibility and (2.3) we obtain

$$
\widehat{\vec{P}}(\vec{x}, \omega)=\varepsilon_{0} \frac{\Delta \varepsilon}{1+j \omega \tau} \widehat{\vec{E}}(\vec{x}, \omega)
$$

and finally by inverse Fourier transformation

$$
\vec{P}+\tau \frac{\partial \vec{P}}{\partial t}=\varepsilon_{0}\left(\varepsilon_{s}-\varepsilon_{\infty}\right) \vec{E}
$$

We can now state Maxwell's equations in a Debye dispersive medium. We infer from (2.1) and (2.5) that the magnetic field $\vec{H}$, the electric field $\vec{E}$ and the electric polarization $\vec{P}$ satisfy the following system of equations in $[0, T]$

$$
\left\{\begin{array}{l}
\mu_{0} \frac{\partial \vec{H}}{\partial t}=-\operatorname{curl}(\vec{E}) \\
\varepsilon_{0} \varepsilon_{\infty} \frac{\partial \vec{E}}{\partial t}=\operatorname{curl}(\vec{H})-\frac{\varepsilon_{0}\left(\varepsilon_{s}-\varepsilon_{\infty}\right)}{\tau} \vec{E}-\sigma \vec{E}+\frac{1}{\tau} \vec{P} \\
\frac{\partial \vec{P}}{\partial t}=\frac{\varepsilon_{0}\left(\varepsilon_{s}-\varepsilon_{\infty}\right)}{\tau} \vec{E}-\frac{1}{\tau} \vec{P} .
\end{array}\right.
$$

The boundary conditions are usually of the following type: we introduce the decomposition $\partial \Omega=\Gamma_{m} \cup \Gamma_{a}$ of the boundary of $\Omega$ on which we impose

$$
\left\{\begin{array}{l}
\vec{n} \times \vec{E}=0 \quad \text { on } \Gamma_{m}, \\
\vec{n} \times \vec{E}-\sqrt{\frac{\mu}{\varepsilon}} \vec{n} \times(\vec{H} \times \vec{n})=\vec{n} \times \vec{E}^{i n c}-\sqrt{\frac{\mu}{\varepsilon}} \vec{n} \times\left(\vec{H}^{i n c} \times \vec{n}\right) \quad \text { on } \Gamma_{a},
\end{array}\right.
$$

where $\vec{n}$ denotes the unit outward normal to $\partial \Omega$ and $\left(\vec{E}^{i n c}, \vec{H}^{i n c}\right)$ is a given incident field. The first boundary condition is called metallic (referring to a perfectly conductive surface) while the second one is called absorbing and takes the form of the 
Silver-Müller condition which is a first order approximation of the exact absorbing boundary condition.

To simplify the presentation in the rest of the paper, we assume that $\Gamma_{a}=\emptyset$. However, we note that the analysis carried on in the following can be easily extended to the more general setting that includes both a metallic and an absorbing boundary condition.

REMARK 2.1. One can prove that if the fields are initially divergence free, then the fields are divergence free at any time, see [28]. That is why we do not need to consider the third and fourth equations of (2.1) in the system (2.6).

The question of the existence and uniqueness of solutions of system (2.6) has been studied in [27]. Let us introduce $H(\operatorname{curl}, \Omega)$ the classical subspace of $L^{2}(\Omega)$ fields with curl in $L^{2}(\Omega)$ and $H_{0}(\operatorname{curl}, \Omega)$ the classical subspace of $H(\operatorname{curl}, \Omega)$ fields with zero tangential trace. Let us define $\mathcal{H}_{0}(\Omega)=[H(\operatorname{curl}, \Omega)]^{3} \times\left[H_{0}(\operatorname{curl}, \Omega)\right]^{3} \times\left[L^{2}(\Omega)\right]^{3}$, it is proven in $[27]$ that if we assume that $\left(\vec{H}_{0}, \vec{E}_{0}, \vec{P}_{0}\right)$ belongs to $\mathcal{H}$ then system $(2.6)$ has a unique weak solution belonging to $C\left([0, T], \mathcal{H}_{0}(\Omega)\right)$. From now on, we consider a normalized form of Maxwell's equations (2.6). We introduce the normalized space, time variables and physical fields through the relations

$$
\begin{aligned}
& \widetilde{\vec{x}}=\vec{x}, \quad \widetilde{t}=c_{0} t, \quad \widetilde{\tau}=c_{0} \tau, \quad \tilde{\sigma}=Z_{0} \sigma, \\
& \widetilde{\vec{E}}=\vec{E}, \quad \widetilde{\vec{H}}=Z_{0} \vec{H} \quad \text { and } \quad \widetilde{\vec{P}}=\vec{P} / \varepsilon_{0},
\end{aligned}
$$

where $c_{0}=1 / \sqrt{\varepsilon_{0} \mu_{0}}$ is the speed of light in vacuum and $Z_{0}=\sqrt{\mu_{0} / \varepsilon_{0}}$ is the free space intrinsic impedance. For convenience of presentation we omit in the sequel the " " notation except for $\widetilde{\tau}$ to avoid any ambiguity since $\tau$ will denote an element of a mesh, and we denote the relative magnetic permeability, $\mu_{r}$, by $\mu$ and use the notation $\Delta \varepsilon=\varepsilon_{s}-\varepsilon_{\infty}$. We can now write Maxwell's equations as

$$
\left\{\begin{array}{l}
\mu \frac{\partial \vec{H}}{\partial t}=-\operatorname{curl}(\vec{E}) \\
\varepsilon_{\infty} \frac{\partial \vec{E}}{\partial t}=\operatorname{curl}(\vec{H})-\frac{\Delta \varepsilon}{\widetilde{\tau}} \vec{E}-\sigma \vec{E}+\frac{1}{\widetilde{\tau}} \vec{P} \\
\frac{\partial \vec{P}}{\partial t}=\frac{\Delta \varepsilon}{\widetilde{\tau}} \vec{E}-\frac{1}{\widetilde{\tau}} \vec{P}
\end{array}\right.
$$

3. Locally implicit DGTD method for Maxwell's equations in dispersive media. In this section Maxwell's equations (2.7) are discretized in space according to a DG formulation then we adapt the locally implicit time integration method from [12] with the additional ADE and analyze its stability, via an energy approach which provides a rigorous stability criterion.

3.1. Semi-discretization in space by the DG method. As in [27] we write the semi-discrete system of (2.7) using a DG method formulated on simplicial meshes following the approach of [12]. From now on we assume that $\Omega$ is a bounded convex polygonal domain, let $\Omega_{h}$ be a partition of $\Omega$ into a set of $N_{h}$ tetrahedra $\tau_{i}$ of size $h_{i}$ with boundary $\partial \tau_{i}$. We assume that this mesh is shape regular. By convention $h$ denotes the maximum diameter of the (non-uniform) grid elements. An internal face is denoted by $a_{i k}=\tau_{i} \cap \tau_{k}$, for $i \neq k$ and $\vec{n}_{i k}$ is the unitary normal vector oriented from $\tau_{i}$ to $\tau_{k}$. For the boundary interface, the index $k$ corresponds to a fictitious element outside the domain. We denote by $\nu_{i}$ the set of indices of the elements which 
have a common interface with $\tau_{i}$ with the following decomposition $\nu_{i}=\nu_{i}^{i} \cup \nu_{i}^{m}$, with $\nu_{i}^{i}=\left\{k \in \nu_{i}: a_{i k}\right.$ is an internal interface $\}$ and $\nu_{i}^{m}=\left\{k \in \nu_{i}: a_{i k} \in \Gamma_{m}\right\}$. As with a finite element method for the given partition $\Omega_{h}$ we seek approximate electric and magnetic fields and electric polarization $\left(\vec{E}_{h}, \vec{H}_{h}, \vec{P}_{h}\right)$ of $(\vec{E}, \vec{H}, \vec{P})$ solution of (2.7) in a subspace $V_{h}$. We introduce $\mathbb{P}_{p_{i}}\left(\tau_{i}\right)$ the space of polynomial functions of degree at most $p_{i}$ inside the element $\tau_{i}$ and define the following finite dimensional subspace

$$
V_{h}=\left\{\vec{v}=\left(v_{1}, v_{2}, v_{3}\right)^{T} \in\left[L^{2}(\Omega)\right]^{3}, v_{k \mid \tau_{i}} \in \mathbb{P}_{p_{i}}\left(\tau_{i}\right), \quad \forall k, \quad \forall \tau_{i} \in \Omega_{h}\right\} .
$$

Following again the DG approach introduced in [27], inside each finite element $\tau_{i}$, the local electric, magnetic fields and electric polarization $\left(\left.\vec{E}_{h}\right|_{\tau_{i}},\left.\vec{H}_{h}\right|_{\tau_{i}},\left.\vec{P}_{h}\right|_{\tau_{i}}\right)=$ $\left(\vec{E}_{i}, \vec{H}_{i} \vec{P}_{i}\right)$ are expressed as linear combination of linearly independent basis vector $\Phi_{i l}, 1 \leq l \leq 3 d_{i}$,

$$
\begin{aligned}
\vec{E}_{i}(\vec{x}, t) & =\sum_{l=1}^{3 d_{i}} E_{i l}(t) \Phi_{i l}(\vec{x}), \vec{H}_{i}(\vec{x}, t)=\sum_{l=1}^{3 d_{i}} H_{i l}(t) \Phi_{i l}(\vec{x}), \\
\vec{P}_{i}(\vec{x}, t) & =\sum_{l=1}^{3 d_{i}} P_{i l}(t) \Phi_{i l}(\vec{x})
\end{aligned}
$$

where $d_{i}$ denotes the local number of degrees of freedom associated to the interpolation degree $p_{i}$ in $\tau_{i}$, i.e. $d_{i}=\left(p_{i}+1\right)\left(p_{i}+2\right)\left(p_{i}+3\right) / 6$ and $E_{i l}, H_{i l}, P_{i l}$ reflect nodal values of $\vec{E}_{i}, \vec{H}_{i}$ and $\vec{P}_{i}$, respectively. We now derive the DG spatial discretization by establishing local variational formulations on each element of the space grid. For each $\tau_{i}, \mu_{i}, \varepsilon_{i \infty}, \varepsilon_{i s}, \widetilde{\tau}_{i}$ and $\sigma_{i}$, denote respectively the local magnetic permeability of the medium, the local infinite frequency relative permittivity, the local static relative permittivity, the local Debye relaxation time constant and the conduction coefficient, which are assumed constant inside the element $\tau_{i}$. We still use the notation $\Delta \epsilon_{i}=$ $\varepsilon_{i s}-\varepsilon_{i \infty}$. Dot-multiplying (2.7) by any given vector $\Phi$ belonging to $\operatorname{Span}\left(\Phi_{i j}, 1 \leq\right.$ $\left.j \leq 3 d_{i}\right)$, integrating over each element $\tau_{i}$, integrating by part and finally replacing the exact fields $(\vec{E}, \vec{H}, \vec{P})$ by the approximate fields $\left(\vec{E}_{h}, \vec{H}_{h}, \vec{P}_{h}\right)$, yields

$$
\left\{\begin{aligned}
\int_{\tau_{i}} \mu_{i} \Phi \cdot \partial_{t} \vec{H}_{h} d \vec{x} & +\int_{\tau_{i}} \operatorname{curl} \Phi \cdot \vec{E}_{h} d \vec{x}-\int_{\partial \tau_{i}} \Phi \cdot\left(\vec{E}_{h} \times \vec{n}\right) d s=0 \\
\int_{\tau_{i}} \varepsilon_{i \infty} \Phi \cdot \partial_{t} \vec{E}_{h} d \vec{x} & -\int_{\tau_{i}} \operatorname{curl} \Phi \cdot \vec{H}_{h} d \vec{x}+\int_{\partial \tau_{i}} \Phi \cdot\left(\vec{H}_{h} \times \vec{n}\right) d s \\
& +\int_{\tau_{i}} \sigma_{i} \Phi \cdot \vec{E}_{h} d \vec{x}-\int_{\tau_{i}} \frac{\Delta \varepsilon_{i}}{\widetilde{\tau}_{i}} \Phi \cdot \vec{E}_{h} d \vec{x}+\int_{\tau_{i}} \frac{\Phi}{\widetilde{\tau}_{i}} \cdot \vec{P}_{h} d \vec{x}=0 \\
\int_{\tau_{i}} \Phi \cdot \partial_{t} \vec{P}_{h} d \vec{x} & -\int_{\tau_{i}} \frac{\Delta \varepsilon_{i}}{\widetilde{\tau}_{i}} \Phi \cdot \vec{E}_{h} d \vec{x}+\int_{\tau_{i}} \frac{\Phi}{\widetilde{\tau}_{i}} \cdot \vec{P}_{h} d \vec{x}=0
\end{aligned}\right.
$$

One of the main features of the DG approach is that the approximate fields are allowed to be discontinuous across element boundaries. Then, for such discontinuous fields, we must define an approximate trace (i.e. a numerical trace) to evaluate the integrals over $\partial \tau_{i}$. In this study, we choose to use a centered approximation

$$
\forall i, \forall k \in \nu_{i},\left.\quad \vec{E}_{h}\right|_{a_{i k}}=\frac{\left.\vec{E}_{i}\right|_{a_{i k}}+\left.\vec{E}_{k}\right|_{a_{i k}}}{2} \text { and }\left.\quad \vec{H}_{h}\right|_{a_{i k}}=\frac{\left.\vec{H}_{i}\right|_{a_{i k}}+\left.\vec{H}_{k}\right|_{a_{i k}}}{2} .
$$


Now using $\left(\left.\vec{E}_{h}\right|_{\tau_{i}},\left.\vec{H}_{h}\right|_{\tau_{i}},\left.\vec{P}_{h}\right|_{\tau_{i}}\right)=\left(\vec{E}_{i}, \vec{H}_{i}, \vec{P}_{i}\right)$ for volume integrals, evaluating the surface integrals in (3.2) with the centered numerical flux (3.3) and re-integrating by parts, yields

$$
\left\{\begin{aligned}
\int_{\tau_{i}} \mu_{i} \Phi \cdot \partial_{t} \vec{H}_{i} d \vec{x}= & -\frac{1}{2} \int_{\tau_{i}}\left(\operatorname{curl} \Phi \cdot \vec{E}_{i}+\operatorname{curl} \vec{E}_{i} \cdot \Phi\right) d \vec{x}+ \\
\int_{\tau_{i}} \varepsilon_{i \infty} \Phi \cdot \partial_{t} \vec{E}_{i} d \vec{x}= & \frac{1}{2} \sum_{k \in \nu_{i}} \int_{\tau_{i k}} \Phi \cdot\left(\vec{E}_{k} \times \vec{n}_{i k}\right) d s \\
& \frac{1}{2} \sum_{k \in \nu_{i}} \int_{a_{i k}} \Phi \cdot\left(\vec{H}_{k} \times \vec{n}_{i k}\right) d s-\int_{\tau_{i}} \sigma_{i} \Phi \cdot \vec{E}_{i} d \vec{x}- \\
& \int_{\tau_{i}} \frac{\Delta \varepsilon_{i}}{\widetilde{\tau}_{i}} \Phi \cdot \vec{E}_{i} d \vec{x}+\int_{\tau_{i}} \frac{\Phi}{\widetilde{\tau}_{i}} \cdot \vec{P}_{i} d \vec{x} \\
\int_{\tau_{i}} \Phi \cdot \partial_{t} \vec{P}_{i} d \vec{x}= & \int_{\tau_{i}} \frac{\Delta \varepsilon_{i}}{\widetilde{\tau}_{i}} \Phi \cdot \vec{E}_{i} d \vec{x}-\int_{\tau_{i}} \frac{\Phi}{\widetilde{\tau}_{i}} \cdot \vec{P}_{i} d \vec{x} .
\end{aligned}\right.
$$

Then for each face on the boundary of $\Omega_{h}, a_{i k}$, the trace of a fictitious neighboring element is needed for the computation of the numerical flux, we impose $\left.\vec{E}_{k}\right|_{a_{i k}}=$ $-\left.\vec{E}_{i}\right|_{a_{i k}}$ and $\left.\vec{H}_{k}\right|_{a_{i k}}=\left.\vec{H}_{i}\right|_{a_{i k}}$. The system of equations (3.4) can be written in terms of scalar unknowns. According to the decomposition (3.1) of the local electric, magnetic fields and electric polarization $\left(\vec{E}_{i}, \vec{H}_{i}, \vec{P}_{i}\right)$, denoting the column vectors $\left(E_{i l}\right)_{1<l<3 d_{i}}$, $\left(H_{i l}\right)_{1 \leq l \leq 3 d_{i}}$ and $\left(P_{i l}\right)_{1 \leq l \leq 3 d_{i}}$ by $E_{i}, H_{i}$ and $P_{i}$, respectively, and replacing $\Phi$ by the basis vectors $\left(\Phi_{i j}\right)_{1 \leq j \leq 3 d_{i}}$, we obtain the equivalent system

$$
\left\{\begin{aligned}
M_{i}^{\mu} \partial_{t} H_{i} & =-K_{i} E_{i}+\sum_{k \in \nu_{i}} S_{i k} E_{k} \\
M_{i}^{\varepsilon} \partial_{t} E_{i} & =K_{i} H_{i}-\sum_{k \in \nu_{i}} S_{i k} H_{k}-D_{i} E_{i} \\
& -M_{i}^{\Delta \varepsilon / \widetilde{\tau}} E_{i}+M_{i}^{1 / \widetilde{\tau}} P_{i} \\
M_{i}^{1} \partial_{t} P_{i} & =M_{i}^{\Delta \varepsilon / \widetilde{\tau}} E_{i}-M_{i}^{1 / \widetilde{\tau}} P_{i}
\end{aligned}\right.
$$

with the following matrices : $M_{i}^{\varsigma}(\varsigma$ stands for $\mu, \varepsilon, \Delta \varepsilon / \widetilde{\tau}$ or $1 / \widetilde{\tau}$ ) are the symmetric, positive definite mass matrices

$$
\left(M_{i}^{\varsigma}\right)_{j l}=\int_{\tau_{i}} \Phi_{i j}^{T} \varsigma_{i} \Phi_{i l} d \vec{x} \quad\left(1 \leq j, l \leq 3 d_{i}\right),
$$

$K_{i}$ is the symmetric stiffness matrix

$$
\left(K_{i}\right)_{j l}=\frac{1}{2} \int_{\tau_{i}}\left(\Phi_{i j}^{T} \operatorname{curl} \Phi_{i l}+\Phi_{i l}^{T} \operatorname{curl} \Phi_{i j}\right) d \vec{x} \quad\left(1 \leq j, l \leq 3 d_{i}\right),
$$

$S_{i k}$ are the rectangular interface matrices

$$
\left(S_{i k}\right)_{j l}=\frac{1}{2} \int_{a_{i k}} \Phi_{i j}^{T}\left(\Phi_{k l} \times \vec{n}_{i k}\right) d s \quad\left(1 \leq j \leq 3 d_{i}, 1 \leq l \leq 3 d_{k}\right) .
$$

$D_{i}$ is the symmetric, positive semi-definite conduction matrix

$$
\left(D_{i}\right)_{j l}=\int_{\tau_{i}} \Phi_{i j}^{T} \sigma_{i} \Phi_{i l} d \vec{x} \quad\left(1 \leq j, l \leq 3 d_{i}\right) .
$$


Taking into account the trace of fictitious neighboring elements and using the previous decomposition $\nu_{i}=\nu_{i}^{i} \cup \nu_{i}^{m}$, we can rewrite the local system of ODEs (3.5) as

$$
\left\{\begin{array}{l}
M_{i}^{\mu} \partial_{t} H_{i}=-K_{i} E_{i}+\sum_{k \in \nu_{i}^{i}} S_{i k} E_{k}-\sum_{k \in \nu_{i}^{m}} S_{i k}^{m} E_{i} \\
M_{i}^{\varepsilon_{\infty}} \partial_{t} E_{i}=K_{i} H_{i}-\sum_{k \in \nu_{i}^{i}} S_{i k} H_{k}+\sum_{k \in \nu_{i}^{m}} S_{i k}^{m} H_{i}-D_{i} E_{i}-M_{i}^{\Delta \varepsilon / \widetilde{\tau}} E_{i}+M_{i}^{1 / \widetilde{\tau}} P_{i} \\
M_{i}^{1} \partial_{t} P_{i}=M_{i}^{\Delta \varepsilon / \widetilde{\tau}} E_{i}-M_{i}^{1 / \widetilde{\tau}} P_{i},
\end{array}\right.
$$

with $\left(S_{i k}^{m}\right)_{j l}=\frac{1}{2} \int_{a_{i k}} \Phi_{i j}^{T}\left(\Phi_{i l} \times \vec{n}_{i k}\right) d s \quad\left(1 \leq j, l \leq 3 d_{i}\right)$. These set of local semidiscrete systems (3.6) for each finite element $\tau_{i}$ can be transformed into a global system. Gathering all electric, magnetic and electric polarization unknowns in column vectors of size $d=\sum_{i=1}^{N_{h}} d_{i}$, denoted $E, H$ and $P$, respectively, we write

$$
\left\{\begin{array}{l}
M^{\mu} \partial_{t} H=-K E+A E-B E \\
M^{\varepsilon} \partial_{t} E=K H-A H+B H-D E-M^{\Delta \varepsilon / \widetilde{\tau}} E+M^{1 / \widetilde{\tau}} P, \\
M^{1} \partial_{t} P=M^{\Delta \varepsilon / \widetilde{\tau}} E-M^{1 / \widetilde{\tau}} P
\end{array}\right.
$$

where $M^{\varsigma}$ and $K$ are $3 d \times 3 d$ block diagonal mass and stiffness matrices with diagonal blocks equal to $M_{i}^{\varsigma}$ and $K_{i}$, respectively. Then the matrices $M^{\varsigma}$ are symmetric, positive definite and $K$ is symmetric. $A$ is a $3 d \times 3 d$ block sparse matrix, whose nonzero blocks are equal to $S_{i k}$ when $a_{i k}$ is an internal interface. From (3.1) and the equality $\vec{n}_{k i}=-\vec{n}_{i k}$ we can check that $S_{k i}=S_{i k}^{T}$ and then $A$ is symmetric

$$
\left(S_{k i}\right)_{l j}=\frac{1}{2} \int_{a_{i k}}-\Phi_{k l}^{T}\left(\Phi_{i j} \times \vec{n}_{i k}\right) d s=\frac{1}{2} \int_{a_{i k}} \Phi_{i j}^{T}\left(\Phi_{k l} \times \vec{n}_{i k}\right) d s=\left(S_{i k}\right)_{j l} .
$$

$B$ is a $3 d \times 3 d$ block diagonal matrix, whose nonzero blocks equal to $S_{i k}^{m}$. In the present case $\left(S_{i k}^{m}\right)_{j l}=-\left(S_{i k}^{m}\right)_{l j}$, then $S_{i k}^{m}=-\left(S_{i k}^{m}\right)^{T}$ and $B$ is skew-symmetric. To simplify the notations and for the rest of this section we assume the different coefficients are constant and introduce the notation $S=K-A-B$, allowing now to drop the $\sim$ on $\tau$ we then obtain the following system

$$
\left\{\begin{array}{l}
\mu M \frac{\partial H}{\partial t}=-S^{T} E \\
\varepsilon_{\infty} M \frac{\partial E}{\partial t}=S H-\frac{\left(\varepsilon_{s}-\varepsilon_{\infty}\right)}{\tau} M E-\sigma M E+\frac{1}{\tau} M P \\
M \frac{\partial P}{\partial t}=\frac{\left(\varepsilon_{s}-\varepsilon_{\infty}\right)}{\tau} M E-\frac{1}{\tau} M P
\end{array}\right.
$$

Now we use the Cholesky factorization of the mass matrix $M=L_{M} L_{M}^{T}$, where $L_{M}$ is a triangular matrix, and we introduce the change of variables $\widetilde{E}=L_{M}^{T} E, \widetilde{H}=L_{M}^{T} H$ 
and $\widetilde{P}=L_{M}^{T} P$ in $(3.7)$, hence

$$
\left\{\begin{array}{l}
\mu \frac{\partial \widetilde{H}}{\partial t}=-\widetilde{S}^{T} \widetilde{E} \\
\varepsilon_{\infty} \frac{\partial \widetilde{E}}{\partial t}=\widetilde{S} \widetilde{H}-\frac{\left(\varepsilon_{s}-\varepsilon_{\infty}\right)}{\tau} \widetilde{E}-\sigma \widetilde{E}+\frac{1}{\tau} \widetilde{P} \\
\frac{\partial \widetilde{P}}{\partial t}=\frac{\left(\varepsilon_{s}-\varepsilon_{\infty}\right)}{\tau} \widetilde{E}-\frac{1}{\tau} \widetilde{P}
\end{array}\right.
$$

where $\widetilde{S}=L_{M}^{-1} S\left(L_{M}^{-1}\right)^{T}$. For convenience of presentation we again omit from now on the notation tilde in (3.8) and we focus now on the stability of this semi-discrete scheme. In the rest of the paper we denote $\langle\cdot, \cdot\rangle$ the discrete $L^{2}$ inner product on $\mathbb{R}^{d}$ and $\|\cdot\|_{2}$ the corresponding norm. Let $\left(H_{h}, E_{h}, P_{h}\right)$ be the solution of the semi-discrete system (3.8), defining the semi-discrete energy by

$$
\mathcal{E}_{h}=\frac{1}{2}\left(\mu\left\|H_{h}\right\|_{2}^{2}+\varepsilon_{\infty}\left\|E_{h}\right\|_{2}^{2}+\frac{1}{\left(\varepsilon_{s}-\varepsilon_{\infty}\right)}\left\|P_{h}\right\|_{2}^{2}\right),
$$

as in [27], we can prove that $\mathcal{E}_{h}$ is a decreasing function in time so that $\mathcal{E}_{h}(t) \leq \mathcal{E}_{h}(0)$, showing stability in the discrete $L^{2}$ sense on a fixed mesh.

3.2. Time integration methods. Adapting the locally implicit time integration method from [43] with an additional ADE we introduce the implicit-explicit time integration scheme for the semi-discrete system (3.8)

$$
\left\{\begin{aligned}
\mu \frac{H^{n+1 / 2}-H^{n}}{\Delta t / 2} & =-S^{T} E^{n}, \\
\varepsilon_{\infty} \frac{E^{n+1}-E^{n}}{\Delta t} & =S_{0} H^{n+1 / 2}+\frac{1}{2} S_{1}\left(H^{n+1}+H^{n}\right) \\
& -\frac{\left(\varepsilon_{s}-\varepsilon_{\infty}\right)}{2 \tau}\left(E^{n+1}+E^{n}\right) \\
& -\frac{1}{2} \sigma\left(E^{n+1}+E^{n}\right)+\frac{1}{2 \tau}\left(P^{n+1}+P^{n}\right), \\
\frac{P^{n+1}-P^{n}}{\Delta t} & =\frac{\left(\varepsilon_{s}-\varepsilon_{\infty}\right)}{2 \tau}\left(E^{n+1}+E^{n}\right)-\frac{1}{2 \tau}\left(P^{n+1}+P^{n}\right), \\
\mu \frac{H^{n+1}-H^{n+1 / 2}}{\Delta t / 2} & =-S^{T} E^{n+1},
\end{aligned}\right.
$$

where $S=S_{0}+S_{1}$ is a matrix splitting. The method is implicit in $S_{1}$ and explicit in $S_{0}$. For $S_{0}=0$ we recover the second order leap-frog scheme and for $S_{1}=0$ the second order Crank-Nicolson scheme both written in a three-stage form. We adopt the splitting defined in [43], i.e. $S_{1}=S S_{H}$, where $S_{H}$ is a diagonal matrix of dimension the length of $H$ defined by

$$
\left(S_{H}\right)_{j j}=\left\{\begin{array}{l}
0, \text { component } H_{j} \text { of } H \text { to be treated explicitly, } \\
1, \text { component } H_{j} \text { of } H \text { to be treated implicitly. }
\end{array}\right.
$$

From the third and the fourth equations of (3.9) we express the electric polarization $P^{n+1}$ and the magnetic field $H^{n+1}$ as

$$
P^{n+1}=\left(\frac{2 \tau-\Delta t}{2 \tau+\Delta t}\right) P^{n}+\left(\frac{\Delta t}{2 \tau+\Delta t}\right)\left(\varepsilon_{s}-\varepsilon_{\infty}\right)\left(E^{n+1}+E^{n}\right),
$$


and

$$
H^{n+1}=H^{n+1 / 2}-\frac{1}{\mu} \frac{\Delta t}{2} S^{T} E^{n+1} .
$$

Now we substitute these expressions into the second stage of (3.9)

$$
\begin{aligned}
\varepsilon_{\infty} \frac{E^{n+1}-E^{n}}{\Delta t} & =S_{0} H^{n+1 / 2}+\frac{1}{2} S_{1}\left(H^{n}+H^{n+1}\right) \\
& -\frac{1}{2 \tau}\left(\varepsilon_{s}-\varepsilon_{\infty}\right) E^{n}-\frac{1}{2} \sigma E^{n}+\frac{1}{2 \tau}\left(\frac{\Delta t}{2 \tau+\Delta t}\right)\left(\varepsilon_{s}-\varepsilon_{\infty}\right) E^{n} \\
& -\frac{1}{2 \tau}\left(\varepsilon_{s}-\varepsilon_{\infty}\right) E^{n+1}-\frac{1}{2} \sigma E^{n+1} \\
& +\frac{1}{2 \tau}\left(\frac{\Delta t}{2 \tau+\Delta t}\right)\left(\varepsilon_{s}-\varepsilon_{\infty}\right) E^{n+1} \\
& -\frac{1}{\mu} \frac{\Delta t}{4} S_{1} S^{T} E^{n+1}+\frac{1}{2 \tau} P^{n}+\frac{1}{2 \tau}\left(\frac{2 \tau-\Delta t}{2 \tau+\Delta t}\right) P^{n} .
\end{aligned}
$$

Note that $S_{1} S^{T}=S_{1} S_{1}^{T}$, the second stage (3.10) is then equivalent to the following linear system

$$
\mathcal{M} E^{n+1}=b_{n},
$$

where

$$
\begin{aligned}
\mathcal{M} & =\left[1-\frac{1}{\varepsilon_{\infty}} \frac{\Delta t}{2 \tau}\left(\frac{\Delta t}{2 \tau+\Delta t}\right)\left(\varepsilon_{s}-\varepsilon_{\infty}\right)+\frac{1}{\varepsilon_{\infty}} \frac{\Delta t}{2 \tau}\left(\varepsilon_{s}-\varepsilon_{\infty}\right)+\frac{1}{\varepsilon_{\infty}} \frac{\Delta t}{2} \sigma\right] I \\
& +\frac{1}{\varepsilon_{\infty} \mu} \frac{\Delta t^{2}}{4} S_{1} S_{1}^{T}
\end{aligned}
$$

and

$$
\begin{aligned}
b_{n} & =\left[1+\frac{1}{\varepsilon_{\infty}} \frac{\Delta t}{2 \tau}\left(\frac{\Delta t}{2 \tau+\Delta t}\right)\left(\varepsilon_{s}-\varepsilon_{\infty}\right)-\frac{1}{\varepsilon_{\infty}} \frac{\Delta t}{2 \tau}\left(\varepsilon_{s}-\varepsilon_{\infty}\right)-\frac{1}{\varepsilon_{\infty}} \frac{\Delta t}{2} \sigma\right] E^{n} \\
& +\frac{1}{\varepsilon_{\infty}} \Delta t S_{0} H^{n+1 / 2}+\frac{1}{\varepsilon_{\infty}} \frac{\Delta t}{2} S_{1}\left(H^{n+1 / 2}+H^{n}\right) \\
& +\frac{1}{\varepsilon_{\infty}} \frac{\Delta t}{2 \tau}\left[1+\left(\frac{2 \tau-\Delta t}{2 \tau+\Delta t}\right)\right] P^{n}
\end{aligned}
$$

Hence we can write the locally implicit scheme (3.9) as

$$
\left\{\begin{aligned}
H^{n+1 / 2} & =H^{n}-\frac{1}{\mu} \frac{\Delta t}{2} S^{T} E^{n} \\
\mathcal{M} E^{n+1} & =b_{n}, \\
P^{n+1} & =\left(\frac{2 \tau-\Delta t}{2 \tau+\Delta t}\right) P^{n}+\left(\frac{\Delta t}{2 \tau+\Delta t}\right)\left(\varepsilon_{s}-\varepsilon_{\infty}\right)\left(E^{n+1}+E^{n}\right), \\
H^{n+1} & =H^{n+1 / 2}-\frac{1}{\mu} \frac{\Delta t}{2} S^{T} E^{n+1},
\end{aligned}\right.
$$

where the matrix $\mathcal{M}$ and the right hand side $b_{n}$ are defined by (3.12) and (3.13), respectively. 
3.3. Stability of the fully discrete scheme on a fixed mesh. In this section we are interested in the stability of the fully discrete locally implicit scheme (3.9). The derivations in the remainder of this section follow an energy approach which provides a rigorous criterion for stability. We first exhibit a discrete energy, which is a quadratic form of the numerical unknowns, we then show that this energy is a positive definite quadratic form and finally we prove that this energy is decreasing, which achieves the stability analysis. We define the discrete electromagnetic energy, denoted $\mathcal{E}_{n}$, as

$$
\mathcal{E}_{n}=\frac{1}{2}\left(\mu\left\|H^{n}\right\|_{2}^{2}+\varepsilon_{\infty}\left\|E^{n}\right\|_{2}^{2}+\frac{1}{\left(\varepsilon_{s}-\varepsilon_{\infty}\right)}\left\|P^{n}\right\|_{2}^{2}-\frac{\Delta t^{2}}{4 \mu}\left\langle S_{0} S^{T} E^{n}, E^{n}\right\rangle\right),
$$

We first state a condition on the time step $\Delta t$ such that $\mathcal{E}_{n}$ is a positive definite quadratic form.

LEMma 3.1. The quadratic form $\mathcal{E}_{n}$ given by $(3.15)$ is a positive definite quadratic form of the numerical unknowns $H^{n}, E^{n}$ and $P^{n}$ if

$$
\Delta t<\frac{2 \sqrt{\varepsilon_{\infty} \mu}}{\sqrt{\rho\left(S_{0} S_{0}^{T}\right)}},
$$

where $\rho\left(S_{0} S_{0}^{T}\right)$ denotes the spectral radius of $S_{0} S_{0}^{T}$.

Proof. By definition of $S_{0}=S\left(I-S_{H}\right)$ the matrix $S_{0} S^{T}$ is symmetric, since $S_{0} S^{T}=S\left(I-S_{H}\right) S^{T}=S\left(I-S_{H}\right)\left(I-S_{H}\right) S^{T}=S_{0} S_{0}^{T}$. Then, from (3.15) we write the electromagnetic energy as

$$
\begin{aligned}
\mathcal{E}_{n} & =\frac{1}{2}\left(\mu\left\|H^{n}\right\|_{2}^{2}+\varepsilon_{\infty}\left\|E^{n}\right\|_{2}^{2}+\frac{1}{\left(\varepsilon_{s}-\varepsilon_{\infty}\right)}\left\|P^{n}\right\|_{2}^{2}-\frac{\Delta t^{2}}{4 \mu}\left\langle S_{0} S_{0}^{T} E^{n}, E^{n}\right\rangle\right), \\
& =\frac{1}{2}\left(\mu\left\|H^{n}\right\|_{2}^{2}+\varepsilon_{\infty}\left\|E^{n}\right\|_{2}^{2}+\frac{1}{\left(\varepsilon_{s}-\varepsilon_{\infty}\right)}\left\|P^{n}\right\|_{2}^{2}-\frac{\Delta t^{2}}{4 \mu}\left\|S_{0} E^{n}\right\|_{2}^{2}\right) .
\end{aligned}
$$

Furthermore, we have

$$
\left\|S_{0}^{T} E^{n}\right\|_{2} \leq\left\|S_{0}^{T}\right\|_{2}\left\|E^{n}\right\|_{2}=\sqrt{\rho\left(S_{0} S_{0}^{T}\right)}\left\|E^{n}\right\|_{2},
$$

hence

$$
\mathcal{E}_{n} \geq \frac{1}{2}\left(\mu\left\|H^{n}\right\|_{2}^{2}+\left(\varepsilon_{\infty}-\frac{\Delta t^{2}}{4 \mu} \rho\left(S_{0} S_{0}^{T}\right)\right)\left\|E^{n}\right\|_{2}^{2}+\frac{1}{\left(\varepsilon_{s}-\varepsilon_{\infty}\right)}\left\|P^{n}\right\|_{2}^{2}\right),
$$

from which it follows that under the condition (3.16), the discrete energy $\mathcal{E}_{n}$ is a positive definite quadratic form of the numerical unknowns $H^{n}, E^{n}$ and $P^{n}$.

We now prove the following result.

Lemma 3.2. The discrete energy (3.15) is decreasing so that $\mathcal{E}_{n} \leq \mathcal{E}_{0}$.

Proof. From the first and fourth equation of (3.9) we have

$$
\begin{aligned}
H^{n+1 / 2} & =H^{n}-\frac{\Delta t}{2 \mu} S^{T} E^{n}, \\
H^{n+1 / 2} & =H^{n+1}+\frac{\Delta t}{2 \mu} S^{T} E^{n+1} .
\end{aligned}
$$

By substituting half of each expression of (3.17) for $H^{n+1 / 2}$ into the second stage of (3.9) and the first expression of (3.17) into the fourth stage of (3.9), together with 
the final expression of $P^{n+1}$ we obtain

$$
\begin{aligned}
\varepsilon_{\infty}\left(E^{n+1}-E^{n}\right) & =\frac{\Delta t}{2} S\left(H^{n+1}+H^{n}\right)+\frac{\Delta t}{2 \tau}\left(P^{n+1}+P^{n}\right) \\
& -\frac{\left(\varepsilon_{s}-\varepsilon_{\infty}\right) \Delta t}{2 \tau}\left(E^{n+1}+E^{n}\right)-\frac{\Delta t}{2} \sigma\left(E^{n+1}+E^{n}\right) \\
& +\frac{\Delta t^{2}}{4 \mu} S_{0} S^{T}\left(E^{n+1}-E^{n}\right), \\
\frac{1}{\left(\varepsilon_{s}-\varepsilon_{\infty}\right)}\left(P^{n+1}-P^{n}\right) & =\frac{\Delta t}{2 \tau}\left(E^{n+1}+E^{n}\right)-\frac{\Delta t}{2 \tau\left(\varepsilon_{s}-\varepsilon_{\infty}\right)}\left(P^{n+1}+P^{n}\right), \\
\mu\left(H^{n+1}-H^{n}\right) & =-\frac{\Delta t}{2} S^{T}\left(E^{n+1}+E^{n}\right) .
\end{aligned}
$$

Taking inner product with $E^{n+1}+E^{n}, P^{n+1}+P^{n}$ and $H^{n+1}+H^{n}$ yields, respectively

$$
\begin{aligned}
\varepsilon_{\infty}\left(\left\|E^{n+1}\right\|_{2}^{2}-\left\|E^{n}\right\|_{2}^{2}\right) & =\frac{\Delta t}{2}\left\langle S\left(H^{n+1}+H^{n}\right), E^{n+1}+E^{n}\right\rangle \\
& +\frac{\Delta t}{2 \tau}\left\langle P^{n+1}+P^{n}, E^{n+1}+E^{n}\right\rangle \\
& -\frac{\left(\varepsilon_{s}-\varepsilon_{\infty}\right) \Delta t}{2 \tau}\left\|E^{n+1}+E^{n}\right\|_{2}^{2} \\
& -\frac{\Delta t}{2} \sigma\left\|E^{n+1}+E^{n}\right\|_{2}^{2} \\
& +\frac{\Delta t^{2}}{4 \mu}\left\langle S_{0} S^{T}\left(E^{n+1}-E^{n}\right), E^{n+1}+E^{n}\right\rangle, \\
\frac{1}{\left(\varepsilon_{s}-\varepsilon_{\infty}\right)}\left(\left\|P^{n+1}\right\|_{2}^{2}-\left\|P^{n}\right\|_{2}^{2}\right) & =\frac{\Delta t}{2 \tau}\left\langle E^{n+1}+E^{n}, P^{n+1}+P^{n}\right\rangle \\
& -\frac{\Delta t}{2 \tau\left(\varepsilon_{s}-\varepsilon_{\infty}\right)}\left\|P^{n+1}+P^{n}\right\|_{2}^{2}, \\
\mu\left(\left\|H^{n+1}\right\|_{2}^{2}-\left\|H^{n}\right\|_{2}^{2}\right) & =\frac{\Delta t}{2}\left\langle S^{T}\left(E^{n+1}+E^{n}\right), H^{n+1}+H^{n}\right\rangle .
\end{aligned}
$$

We recall that $S_{0} S^{T}=S_{0} S_{0}^{T}$, hence

$$
\begin{aligned}
\left\langle S_{0} S^{T}\left(E^{n+1}-E^{n}\right), E^{n+1}+E^{n}\right\rangle & =\left\langle S_{0} S_{0}^{T}\left(E^{n+1}-E^{n}\right), E^{n+1}+E^{n}\right\rangle, \\
& =\left\langle S_{0} S_{0}^{T} E^{n+1}, E^{n+1}\right\rangle-\left\langle S_{0} S_{0}^{T} E^{n}, E^{n}\right\rangle, \\
& =\left\langle S_{0} S^{T} E^{n+1}, E^{n+1}\right\rangle-\left\langle S_{0} S^{T} E^{n}, E^{n}\right\rangle .
\end{aligned}
$$

Substituting this expression into the first equation of (3.18) and adding the three equations of (3.18) yields

$$
\begin{aligned}
\frac{\mathcal{E}_{n+1}-\mathcal{E}_{n}}{\Delta t} & =-\frac{1}{4 \tau\left(\varepsilon_{s}-\varepsilon_{\infty}\right)}\left\|P^{n+1}-P^{n}\right\|_{2}^{2}+\frac{1}{2 \tau}\left\langle P^{n+1}+P^{n}, E^{n+1}+E^{n}\right\rangle \\
& -\frac{\left(\varepsilon_{s}-\varepsilon_{\infty}\right)}{4 \tau}\left\|E^{n+1}-E^{n}\right\|_{2}^{2}-\frac{1}{4} \sigma\left\|E^{n+1}-E^{n}\right\|_{2}^{2}
\end{aligned}
$$


then

$$
\begin{aligned}
\frac{\mathcal{E}_{n+1}-\mathcal{E}_{n}}{\Delta t} & =-\frac{1}{4 \tau\left(\varepsilon_{s}-\varepsilon_{\infty}\right)}\left\|\left(P^{n+1}+P^{n}\right)-\left(\varepsilon_{s}-\varepsilon_{\infty}\right)\left(E^{n+1}+E^{n}\right)\right\|_{2}^{2} \\
& -\frac{1}{4} \sigma\left\|E^{n+1}-E^{n}\right\|_{2}^{2}, \\
& \leq 0 .
\end{aligned}
$$

This concludes the proof of Lemma 3.2.

3.4. Convergence. In this section we are interested in the PDE convergence of the locally implicit method (3.9). As in [43] we wonder whether the method retains its second-order ODE convergence under stable simultaneous space-time grid refinement $\Delta t \backsim h, h \rightarrow 0$ towards the exact PDE solution. This is not a priori clear due to the component splitting which can introduce order reduction through error constants which grow with $h^{-1}$, for $h \rightarrow 0$, see for example [34]. By applying the method described in [43] and [34], it is possible to prove that the subdivision into coarse and fine elements is not detrimental to the second-order ODE convergence of the method (3.9), under stable simultaneous space-time grid refinement towards the exact underlying PDE solution. This convergence result is given in the following theorem :

TheOREM 3.3. Let $H_{h}(t), E_{h}(t)$ and $P_{h}(t)$ denote the exact solutions of the Maxwell problem in dispersive media under consideration, restricted to the space grid, i.e. the exact solutions of the system of ODEs

$$
\left\{\begin{aligned}
\mu \frac{d}{d t} H_{h}(t) & =-S^{T} E_{h}(t)+\zeta_{h}^{H}(t) \\
\varepsilon_{\infty} \frac{d}{d t} E_{h}(t) & =S H_{h}(t)-\frac{\left(\varepsilon_{s}-\varepsilon_{\infty}\right)}{\tau} E_{h}(t) \\
& -\sigma E_{h}(t)+\frac{1}{\tau} P_{h}(t)+\zeta_{h}^{E}(t), \\
\frac{d}{d t} P_{h}(t) & =\frac{\left(\varepsilon_{s}-\varepsilon_{\infty}\right)}{\tau} E_{h}(t)-\frac{1}{\tau} P_{h}(t)+\zeta_{h}^{P}(t),
\end{aligned}\right.
$$

where $\zeta_{h}^{H}, \zeta_{h}^{E}$ and $\zeta_{h}^{P}$ denote the spatial truncation errors. Assume a Lax-Richtmyer stable space-time grid refinement $\Delta t \sim h, h \rightarrow 0$. On the interval $[0, T]$ the approximations $H^{n}, E^{n}$ and $P^{n}$ of method (3.9) then converge with order two to $H_{h}(t)$, $E_{h}(t)$ and $P_{h}(t)$.

REMARK 3.1. The proof of this theorem can be found in [35] and more recently in [8]. Futhermore, following the previous results and the techniques developed in the recent prepint [17] in which an error analysis of the fully discrete locally implicit $D G D T$ scheme is presented in the case of non-dispersive media, it is also possible to obtain an error analysis in our dispersive case.

4. Numerical results. The simulations discussed in this section have been performed on a workstation equipped with an Intel Xeon $2.40 \mathrm{GHz}$ processor and $16 \mathrm{~GB}$ of RAM memory. The linear system associated to the locally implicit method (3.9) (see (3.11)) is solved using the MUMPS (MUltifrontal Massively Parallel sparse direct Solver) optimized sparse direct solver [1]. We consider the three-dimensional (normalized) Maxwell's equations in Debye dispersive media (2.7) where we add a given source current density. We denote by $\Omega_{h}$ the computational domain and $\Omega_{h}^{\exp }$ the set of tetrahedra that belong to the region where the explicit method is used into the 
implicit-explicit splitting. The critical step size, denoted $\Delta t_{c}$, used in the numerical tests is then given by

$$
\Delta t_{c}=C F L \times \min _{\tau_{i} \in \Omega_{h}^{e x p}}\left(\frac{4 \times V_{i}}{A_{i}}\right),
$$

where $V_{i}$ and $A_{i}$ are the volume and the surface area of tetrahedron $\tau_{i}$, respectively. The values of the $C F L$ number corresponds to the numerical stability, i.e. the limit beyond which we observe a growth of the discrete energy.

4.1. An artificial validation test. We establish an artificial three-dimensional problem to validate the locally implicit DGTD method proposed here for solving the time domain Maxwell's equations in dispersive media with the Debye model. For that purpose, the computational domain is the cube $[0,1]^{3}$ and we first substitute the solution of the propagation of a standing wave in a cubic PEC cavity given by, for a real $\omega$

$$
\vec{H}=\frac{\pi}{\omega} \sin (\omega t)\left(\begin{array}{c}
-\sin (\pi x) \cos (\pi y) \cos (\pi z) \\
2 \cos (\pi x) \sin (\pi y) \cos (\pi z) \\
-\cos (\pi x) \cos (\pi y) \sin (\pi z)
\end{array}\right)
$$

and

$$
\vec{E}=\cos (\omega t)\left(\begin{array}{c}
-\cos (\pi x) \sin (\pi y) \sin (\pi z) \\
0 \\
\sin (\pi x) \sin (\pi y) \cos (\pi z)
\end{array}\right)
$$

into the third equation of (2.7) to obtain the analytic expression of the electric polarization and find

$$
\vec{P}=-\frac{\left(\varepsilon_{s}-\varepsilon_{\infty}\right) \tau}{1+\omega^{2} \tau^{2}}\left[\frac{1}{\tau} \cos (\omega t)+\omega \sin (\omega t)\right]\left(\begin{array}{c}
\cos (\pi x) \sin (\pi y) \sin (\pi z) \\
0 \\
\sin (\pi x) \sin (\pi y) \cos (\pi z)
\end{array}\right) .
$$

Then we substitute the electric field and the polarization into the second equation of (2.7) to obtain the following current density to impose to have an exact solution

$$
\left\{\begin{aligned}
J^{s, x}(\vec{x}, t) & =\cos (\pi x) \sin (\pi y) \sin (\pi z) \times \\
& {\left[\frac{\varepsilon_{s}-\varepsilon_{\infty}}{1+\omega^{2} \tau^{2}}\left(\frac{1}{\tau} \cos (\omega t)+\omega \sin (\omega t)\right)-\left(\frac{\varepsilon_{s}-\varepsilon_{\infty}}{\tau}+\sigma\right) \cos (\omega t)\right] } \\
J^{s, y}(\vec{x}, t) & =0 \\
J^{s, z}(\vec{x}, t) & =\frac{\varepsilon_{s}-\varepsilon_{\infty}}{\tau} \sin (\pi x) \sin (\pi y) \sin (\pi z) \cos (\omega t) \times \\
& {\left[\frac{\varepsilon_{s}-\varepsilon_{\infty}}{1+\omega^{2} \tau^{2}}\left(\frac{1}{\tau} \cos (\omega t)+\omega \sin (\omega t)\right)-\left(\frac{\varepsilon_{s}-\varepsilon_{\infty}}{\tau}+\sigma\right) \cos (\omega t)\right] }
\end{aligned}\right.
$$

the angular frequency is given by $\omega=2 \pi f\left(\mathrm{rad} \cdot \mathrm{s}^{-1}\right)$ with $f=260 \mathrm{MHz}$ and wavelength $\lambda=1.15 \mathrm{~m}$. We impose a metallic boundary condition $\vec{n} \times \vec{E}=0$ on $\partial \Omega$. In our simulations we choose $\mu=\varepsilon_{\infty}=1, \varepsilon_{s}=5, \sigma=0$ and $\tau=9.4$ picoseconds (ps). The total simulation time is set to $T=1.67 \times 10^{-8} \mathrm{~s}$ which corresponds to $T=5 \mathrm{~m}$ (normalized unit). 
We investigate the space-time convergence order (i.e. for a stable simultaneous space-time grid refinement $\Delta t \backsim h, h \rightarrow 0$ ) of the fully explicit method and the locally implicit methods. We measure the maximal $L^{2}$-norm of the error for a sequence of four successively locally refined tetrahedral meshes, see Figure 4.1 for examples of meshes with the identification of the region for implicit treatment.
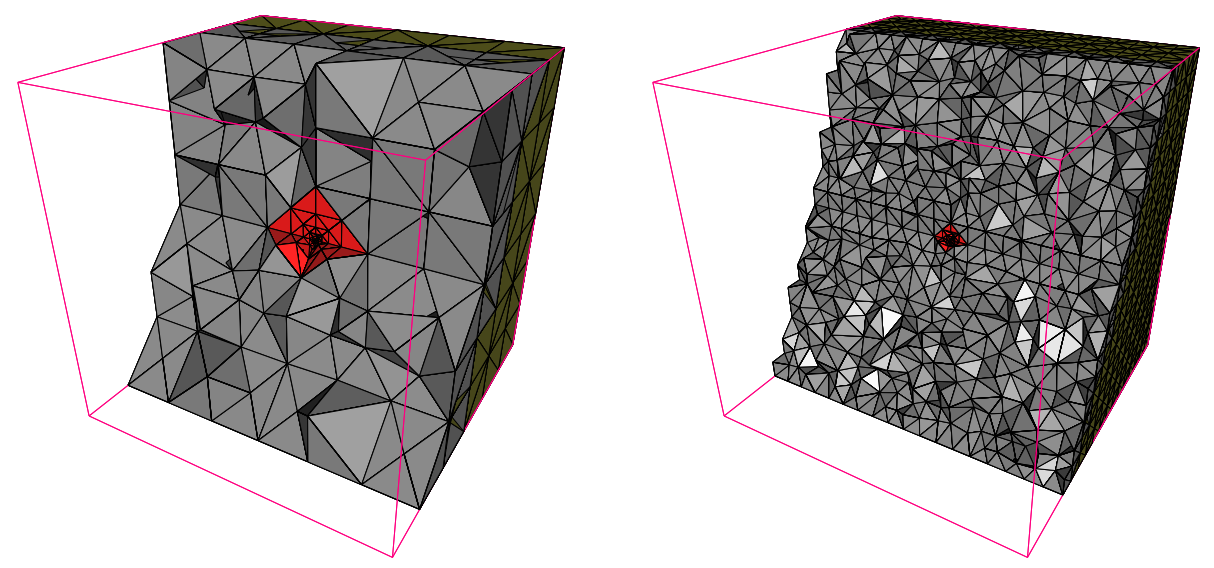

FIG. 4.1. Propagation of a standing wave in a cubic PEC cavity: cross sections of $3 D$ meshes of two locally refined meshes used for the numerical convergence study (implicit treatment: red regions), on left: 2,968 tetraedra, 536 in the implicit region, so approximately $18 \%$ of the elements of the mesh and 635 vertices; on right: 40,616 tetraedra, 536 in the implicit region, so approximately 1, $3 \%$ of the elements of mesh, 7,759 vertices.

We plot this error as a function of $1 / h$, in logarithmic scale, the use of the logarithmic scale allows to visualize the convergence rates as the slopes of the curves. We use the DGTD- $\mathbb{P}_{k}$ method, with $k=1$ or 2 . The obtained results, for both methods, are summarized in Figure 4.2 (left). The results are similar for both time integration methods, which confirm that the subdivision into coarse and fine elements is not detrimental for the convergence order of the locally implicit method. The order of convergence is slightly stronger than the theoretical behavior for $k=1$ (about 1.6 instead of 1.0), while for $k=2$ we obtain the expected order, i.e. the order two. Indeed our DG approach is based on a centered numerical flux for the approximation of the boundary integral term at the interface between neighboring elements, then the convergence rate is $\mathcal{O}\left(h^{k}\right)$ for a $\mathrm{k}$-th interpolation order [12]. The convergence result is slightly weaker than available results for upwind fluxes with $\mathcal{O}\left(h^{k+1}\right)$ convergence rate $[2,36]$. Consequently a suboptimal convergence rate is obtained in the $L^{2}$-norm; nevertheless, this setting yields a non-dissipative numerical scheme which preserves a discrete form of the electromagnetic energy in the case on non-dispersive and nonconducting media, assuming metallic boundary conditions only link in [10] and [12]. We also plot in Figure 4.2 (right) the error as a function of the CPU time. For a given error we can observe the gain in CPU time with the locally implicit approach compared to the fully explicit method. Now we focus on the linear systems to be solved and the efficiency of the locally implicit method. We consider the locally refined tetrahedral mesh composed of 40,616 tetrahedra and 7,756 vertices (Figure 4.1, on right). First, we are interested in the sparsity of the matrix to be inverted and the cost of the factorization step. In Table 4.1 for each matrix of the linear system to solve, we indicate the number of nonzero elements and the fill-in ratio percentage. We also indicate the total size (memory requirement) of all internal data used during nu- 

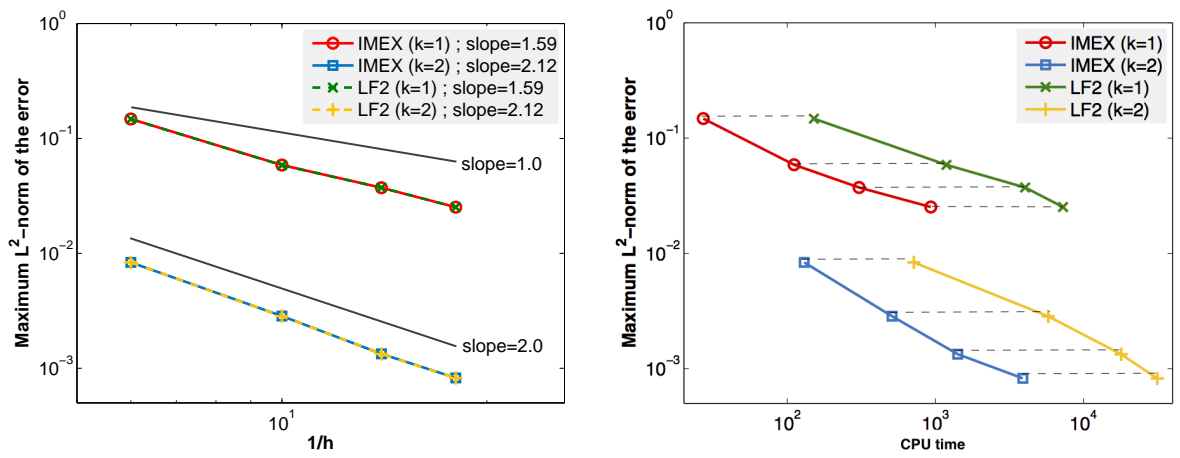

FIG. 4.2. Numerical convergence and maximum error $\left(L^{2}\right.$-norm) in function of final CPU time for the locally implicit and fully explicit $D G T D-\mathbb{P}_{k}$ methods (left - right, respectively).

merical factorization and the total CPU time for analysis and factorization. Note that for the fully implicit scheme based on DGTD- $\mathbb{P}_{1,2}$ methods the number of nonzero is $75,081,406$ and 408, 726, 916 respectively, and will require too much memory to be a reasonable alternative to the fully explicit method. Finally we present some numerical results for the locally implicit method and the fully explicit method. In Table 4.2 we observe that the locally implicit method allows to overcome the step size limitations caused by the local refinement. With implicit-explicit approaches the sizes of the time step are about 34 times larger which yields significant gains of final CPU time, about 8 times lower than the fully explicit case. Regarding the time evolution of the error, Figure 4.3, we observe that the IMEX scheme and the fully explicit scheme based on DGTD- $\mathbb{P}_{1,2}$ methods give similar results.

\begin{tabular}{llllll}
\hline $\mathbb{P}_{k}$ & $\begin{array}{l}\text { Matrix } \\
\text { order }\end{array}$ & $\begin{array}{l}\text { \# nonzero } \\
(\mathrm{nz})\end{array}$ & $\begin{array}{l}\text { Fill-in ratio } \\
\text { (percentage) }\end{array}$ & $\begin{array}{l}\text { RAM size } \\
(\mathrm{MB})\end{array}$ & $\begin{array}{l}\text { CPU time } \\
(\mathrm{s})\end{array}$ \\
\hline $\mathbb{P}_{1}$ & 487,392 & $1,247,266$ & 0.000525 & 210 & 8 \\
$\mathbb{P}_{2}$ & $1,218,480$ & $5,437,770$ & 0.000366 & 937 & 72 \\
\hline
\end{tabular}

TABLE 4.1

Data and factorization of the matrix of the linear system to be solved for the locally implicit methods, with $D G T D-\mathbb{P}_{k}$ methods $(k=1,2)$.

\begin{tabular}{|c|c|c|c|c|c|}
\hline \multirow[b]{2}{*}{$\mathbb{P}_{k}$} & \multicolumn{2}{|c|}{ Fully exp. method } & \multicolumn{2}{|c|}{ Loc. imp. method } & \multirow{2}{*}{$\frac{\mathrm{CPU}_{(\mathrm{LF} 2)}}{\mathrm{CPU}_{(\mathrm{IMEX})}}$} \\
\hline & $\Delta t(\mathrm{~m})$ & CPU time (s) & $\Delta t(\mathrm{~m})$ & CPU time (s) & \\
\hline $\mathbb{P}_{1}$ & $1.1247 \mathrm{e}-4$ & 7285 & $2.6040 \mathrm{e}-3$ & 922 & 7.9 \\
\hline $\mathbb{P}_{2}$ & $7.8267 \mathrm{e}-5$ & 31568 & $4.1502 \mathrm{e}-3$ & 3786 & 8.3 \\
\hline
\end{tabular}

TABLE 4.2

Critical time step size and CPU time for the fully explicit method and the locally implicit method, with DGTD- $\mathbb{P}_{k}$ method $(k=1,2)$. 


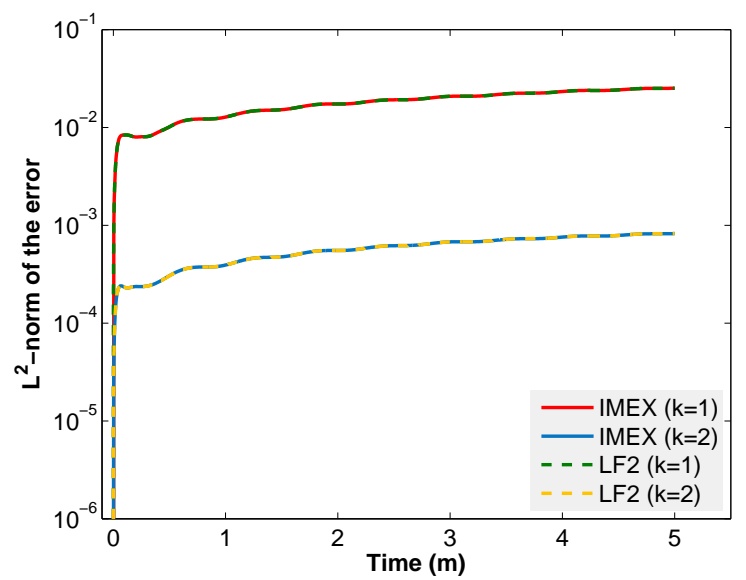

FIG. 4.3. Time evolution of the error ( $L^{2}$-norm) for the locally implicit and fully explicit $D G T D-\mathbb{P}_{k}$ methods.

4.2. Exposure of head tissues to a localized source radiation. We now consider a more realistic test problem in the field of bioelectromagnetics. This problem is of particular importance to the assessment of potential adverse effects resulting from human exposure to electromagnetic fields radiated from wireless systems. More precisely, the goal is to simulate the propagation of an electromagnetic wave in the head tissues when the wave is emitted from a mobile phone antenna. Here, we do not take into account in the numerical modeling a detailed phone structure and simply consider a dipolar source localized near an ear, and concentrate on demonstrating the usefulness of the proposed locally implicit DGTD method (3.9). The geometrical model of the head is heterogeneous and consists of four tissues namely, the skin, the skull, the Cerebro Spinal Fluid (CFS) and the brain. The computational domain is artificially bounded by a sphere on which a Silver-Müller condition is imposed and we use an unstructured tetrahedral mesh consisting of 61,358 vertices and 366, 208 tetrahedra. The surface meshes of the different tissues are shown on Figure 4.4 and the unstructured mesh on Figure 4.5. The Debye model parameters that we have used for the tissues are given in Table 4.3. The numbers have been obtained by applying an appropriate fitting procedure from a set of values for the permittivity of tissues at given frequencies, that have been obtained with a base available at: http://niremf.ifac.cnr.it/tissprop/. 


\begin{tabular}{lllll}
\hline Tissue & Skin & Skull & CSF & Brain \\
\hline$\varepsilon_{\infty}$ & 1.0 & 1.0 & 2.0 & 1.0 \\
$\varepsilon_{s}-\varepsilon_{\infty}$ & 38.0 & 10.5 & 66.0 & 43.0 \\
$\tau(\mathrm{ps})$ & 10.0 & 20.0 & 10.0 & 10.0 \\
$\sigma\left(\mathrm{S} \cdot \mathrm{m}^{-1}\right)$ & 0.7 & 0.1 & 2.0 & 0.7 \\
\hline
\end{tabular}

TABLE 4.3

Debye model parameters for the different tissues.

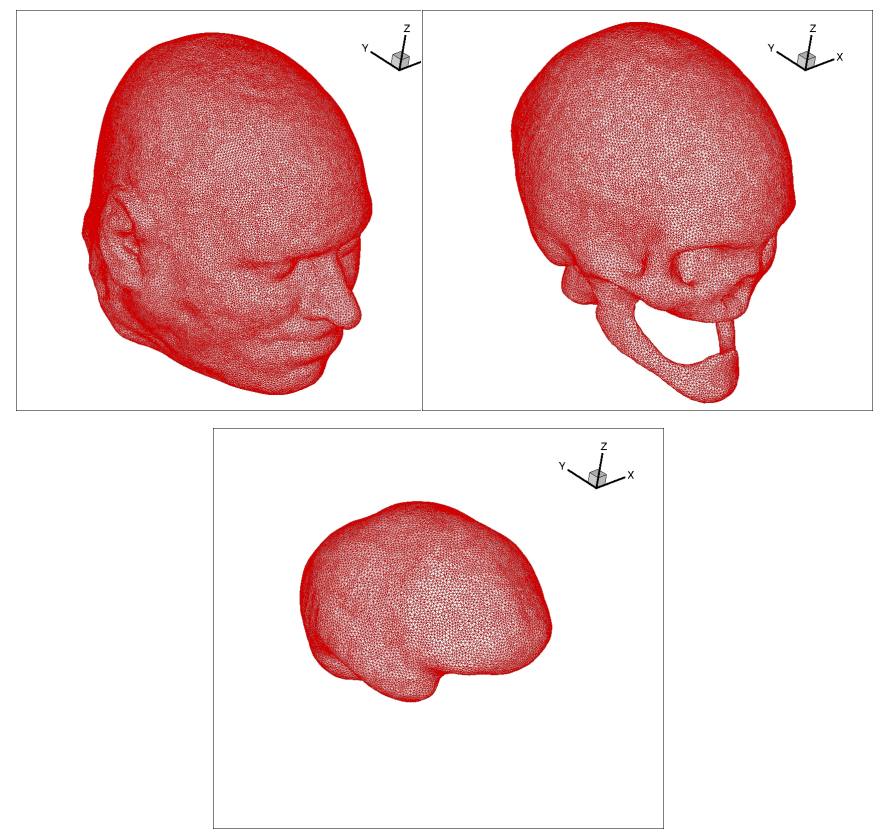

FIG. 4.4. Surface meshes of the skin, the skull and the CSF.
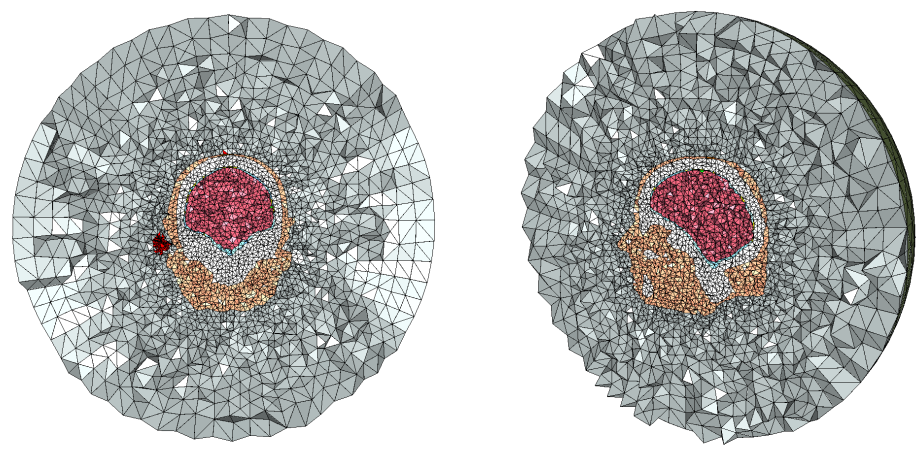

FIG. 4.5. Cross sections of the $3 D$ mesh (61,358 vertices, 366, 208 tetrahedra, implicit treatment: 5092 tetrahedra, dark red region). 
In the present case, there is no incident field and the source term is given by

$$
J^{s, z}(\vec{x}, t)=f_{0} \mathrm{e}^{-\left(t-4 t_{p}\right)^{2} / t_{p}} \sin \left(2 \pi f_{c}\left(t-t_{p}\right)\right) \frac{g(\vec{x})}{\|g(\vec{x})\|},
$$

where $f_{0}$ is the amplitude of the signal, $t_{p}=400 \mathrm{ps}$ and $f_{c}=1.8 \mathrm{GHz}$, and $g$ is a three-dimensional gaussian function with $\left(x_{0}, y_{0}, z_{0}\right)=(-0.100,0.025,-0.015)$ the center of the gaussian spatial support

$$
g(\vec{x})=\mathrm{e}^{-\alpha\left(\left(x-x_{0}\right)^{2}+\left(y-y_{0}\right)^{2}+\left(z-z_{0}\right)^{2}\right)} .
$$

The total physical simulation time is set to $T=10 \times T_{c}$ where $T_{c}=1 / f_{c}$, and a discrete Fourier transform of the electromagnetic field at the frequency $f_{c}$ is computed on the fly. For the simulations we only consider a DGTD- $\mathbb{P}_{1}$ method with the implicit region consisting of 5092 tetrahedra, which represents $1.4 \%$ of the total number of elements. The parameter $\alpha$ in (4.5) has been chosen such that the source term $J^{s, z}$ is strongly localized, which justifies the local refinement around the center of the gaussian, the support of the gaussian $g$ is then strictly included in the implicit region. The localization and the distribution of the elements for the implicit treatment are given in Figure 4.5 and Table 4.4, respectively. Finally, Table 4.5 gives the number of nonzero elements, the fill-in ratio percentage for the matrix of the linear system to be solved, the total size (memory requirement) of all internal data used during numerical factorization and the total CPU time for analysis and factorization.

\begin{tabular}{llllll}
\hline Vacuum & Skin & Skull & CSF & Brain & Total \\
\hline 4209 & 102 & 21 & 720 & 40 & 5092 \\
\hline
\end{tabular}

TABLE 4.4

Exposure of head tissues to a localized source radiation: distribution of elements in the implicit region.

\begin{tabular}{llllll}
\hline $\mathbb{P}_{k}$ & Matrix order & $\begin{array}{l}\text { \# nonzero } \\
(\mathrm{nz})\end{array}$ & $\begin{array}{l}\text { Fill-in ratio } \\
\text { (percentage) }\end{array}$ & $\begin{array}{l}\text { RAM size } \\
(\mathrm{MB})\end{array}$ & $\begin{array}{l}\text { CPU time } \\
(\mathrm{s})\end{array}$ \\
\hline $\mathbb{P}_{1}$ & 4394496 & 15048090 & 0.000078 & 1298 & 95 \\
\hline
\end{tabular}

TABLE 4.5

Exposure of head tissues to a localized source radiation: data and factorization of the matrix of the linear system to be solved for the locally implicit DGTD- $\mathbb{P}_{1}$ method.

A first simulation is performed with the locally implicit DGTD- $\mathbb{P}_{1}$ method (3.9) and a second simulation is realized using the original locally implicit DGTD- $\mathbb{P}_{1}$ method for non-dispersive media with the same localized source term and adopting the appropriate values of the electric permittivity and the electric conductivity for each tissue at the central frequency $f_{c}$. Time evolution of the electric component $E^{z}$ at two selected points is shown on Figure 4.6 for the dispersive and the nondispersive calculations. As expected, the curves obtained for the first point, localized at the center of the gaussian in vacuum are similar. For the second point, localized 

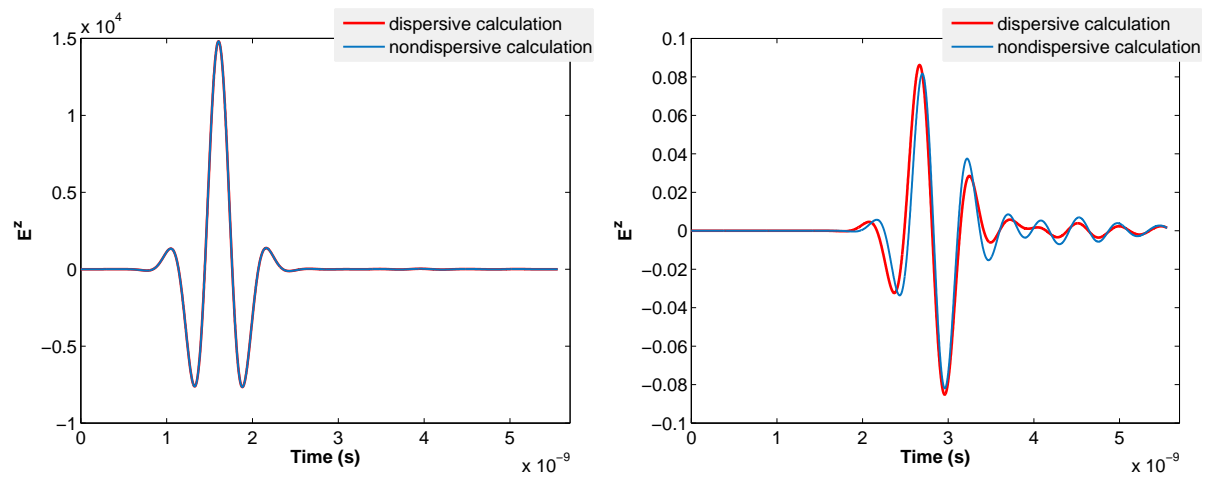

FIG. 4.6. Exposure of head tissues to a localized source radiation: time evolution of the $E^{z}$ component of the electric field at selected spatial locations (center of the gaussian and in the head).

in the head, we can observe the dispersion and a slight attenuation of the signal for the dispersive calculation compared to the non-dispersive calculation.

A third simulation is performed with the fully explicit DGTD- $\mathbb{P}_{1}$ method. We recall that the critical time step size for the stability of the locally implicit method is then about 15.5 times larger than the critical time step size for the stability of the fully explicit method. We recall that the SAR is a measure of the rate at which electric energy is absorbed by the tissues when exposed to a radio-frequency electromagnetic field. It represents the power absorbed per mass of tissue and has units of watts per kilogram $\left(\mathrm{W} \cdot \mathrm{kg}^{-1}\right)$. The SAR is then defined as $\sigma\left|\vec{E}_{\text {four }}\right|^{2} / \rho$, where $\vec{E}_{\text {four }}$ denotes the electric field in the frequency-domain, resulting from the discrete Fourier transform of the temporal field, and $\rho$ is the density which depends on the tissues. We do not observe noticeable differences in the different tissues for the SAR patterns, the obtained results for the locally implicit method (3.9) and the original locally implicit method are globally similar.

On Figures 4.7 we show the contour lines of the local SAR normalized by the maximal local SAR, in logarithmic scale, for the calculations with the locally implicit DGTD- $\mathbb{P}_{1}$ method (3.9). For convenience of presentation we do not show the results obtained with the fully explicit method because they are indistinguishable from that shown in Figure 4.7. Finally, the total simulation times are equal to $6 \mathrm{~h} 38 \mathrm{~min}$ for the locally implicit DGTD- $\mathbb{P}_{1}$ method and $24 \mathrm{~h} 56$ min for the fully explicit DGTD$\mathbb{P}_{1}$ method. Hence, the locally implicit DGTD- $\mathbb{P}_{1}$ method allows a reduction of the computing time by a factor of 3.8 .

5. Conclusion and future works. We have presented a locally implicit DGTD method for simulating the propagation of electromagnetic waves in Debye-type dispersive media in view of biomedical applications. The latter most often involve irregularly shaped structures corresponding to biological tissues. Modeling realistically the interfaces between tissues is particularly important if one is interested in evaluating accurately the impact of field discontinuities on the local SAR distribution. DGTD methods formulated on unstructured tetrahedral meshes are appealing in this context but can be very expensive in terms of computing time when based on a fully explicit time integration scheme. The locally implicit solution strategy considered in this study allows to overcome the restrictive stability condition of global time step 

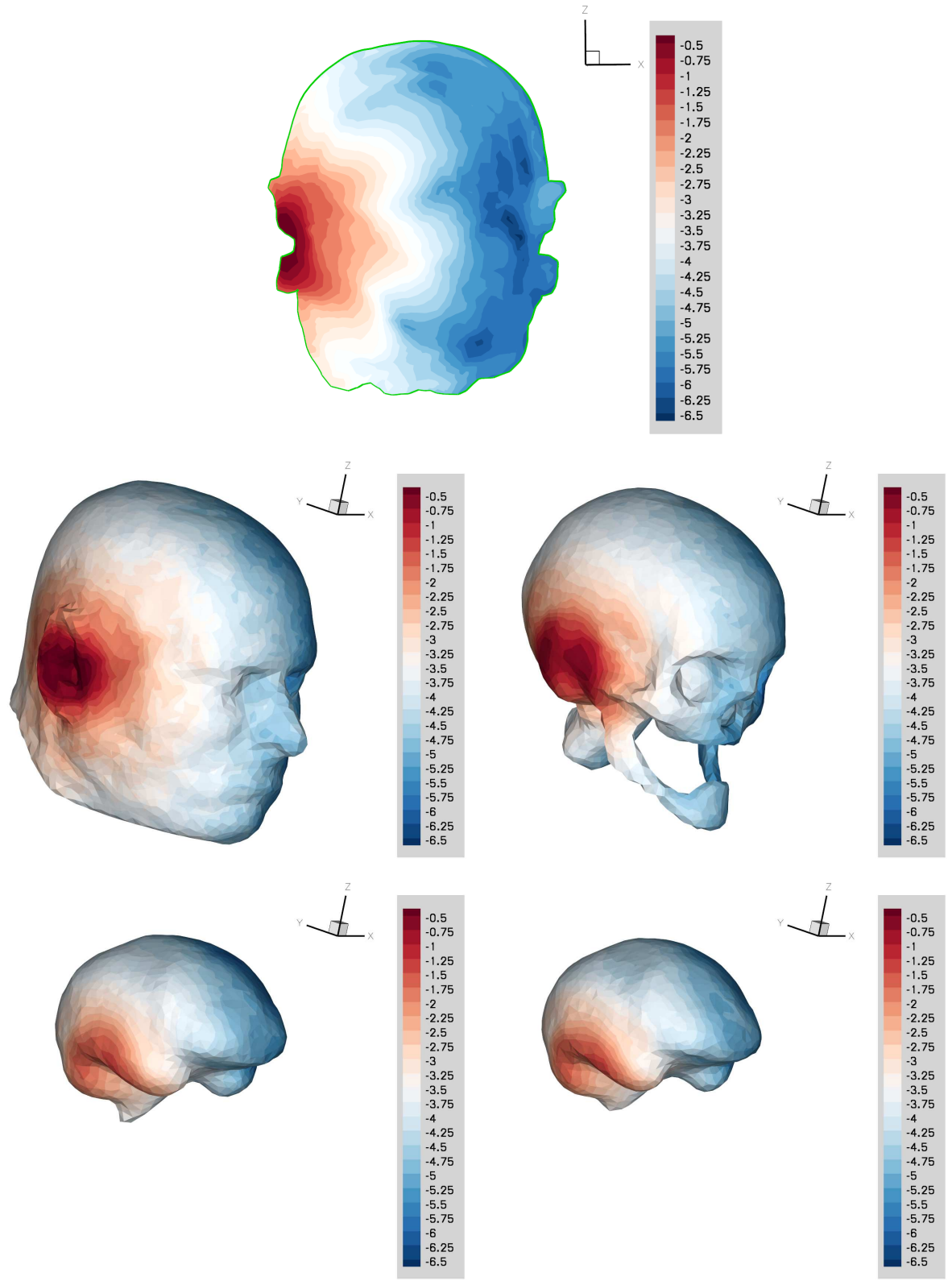

FIG. 4.7. Exposure of head tissues to a localized source radiation: contour lines of the local $S A R$ normalized by the maximal local SAR (logarithmic scale).

explicit schemes at the expense of the inversion of large sparse linear system at each time step. Despite this computational overhead, numerical and performance results obtained here for three-dimensional problems clearly illustrate the benefits of the proposed locally implicit DGTD method. Future works will be concerned with the algorithmic adaptation of the method to parallel computing architectures and the study of high order in time approximations; indeed, composition methods allow to 
reach higher order as seen in [7] for a non-dispersive case. Noteworthy, the method can be easily extended to other dispersion models such as the Drude and Drude-Lorentz models for the interaction of electromagnetic waves with metals at optical frequencies.

\section{REFERENCES}

[1] P. Amestoy, I. Duff, And J.-Y. L'Excellent, Multifrontal parallel distributed symmetric and unsymmetric solvers, Comput. Meth. Appl. Mech. Eng., 184 (2000), pp. 501-520.

[2] B. Cockburn, G. Karniadakis, and C. Shu, Discontinuous Galerkin Methods. Theory, Computation and Applications, vol. 11 of Lecture Notes in Computational Science and Engineering, Springer-Verlag, Berlin, 2000.

[3] B. Cockburn And C. Shu, The Runge-Kutta discontinuous Galerkin method for conservation laws. V. Multidimensional systems, J. Comput. Phys., 141 (1998), pp. 199-224.

[4] _ Special issue on Discontinuous Galerkin methods, J. Sci. Comput., Springer, 22-23 (2005).

[5] M. Converse, E. Bond, S. Hagness, and B. Veen, Ultrawide-band microwave space-time beamforming for hyperthermia treatment of breast cancer: a computational feasibility study, IEEE Trans. Microwave Theory Tech., 52 (2004), pp. 1876-1889.

[6] C. Dawson, Special issue on Discontinuous Galerkin methods, Comput. Meth. App. Mech. Engng., Elsevier, 195 (2006).

[7] S. Descombes, S. Lanteri, And L. Moya, High-order locally implicit time integration strategies in a discontinuous Galerkin method for Maxwell's equations, in Spectral and high order methods for partial differential equations-ICOSAHOM 2012, vol. 95 of Lect. Notes Comput. Sci. Eng., Springer, Cham, 2014, pp. 205-215.

[8] S. Descombes, S. Lanteri, And L. Moya, Temporal convergence analysis of a locally implicit discontinuous galerkin time domain method for electromagnetic wave propagation in dispersive media, tech. rep., Université Nice Sophia Antipolis ; CNRS, 2015. https://hal.archives-ouvertes.fr/hal-01244237.

[9] J. Diaz And M. Grote, Energy conserving explicit local time stepping for second-order wave equations, SIAM J. Sci. Comput., 31 (2009), pp. 1985-2014.

[10] V. Dolean, H. Fahs, L. Fezoui, and S. Lanteri, Locally implicit Discontinuous Galerkin method for time domain electromagnetics, J. Comput. Phys., 229 (2010), pp. 512-526.

[11] E. Fear, P. Meaney, and M. A. Stuchly, Microwaves for breast cancer detection, IEEE Potentials, 22 (2003), pp. 12-18.

[12] L. Fezoui, S. Lanteri, S. Lohrengel, and S. Piperno, Convergence and stability of a Discontinuous Galerkin Time-domain method for the $3 d$ heterogeneous Maxwell equations on unstructured meshes, ESAIM: M2AN, 39 (2005), pp. 1149-1176.

[13] N. Gödel, S. Schomann, T. Warburton, And M. Clemens, Gpu accelerated adamsbashforth multirate discontinuous galerkin fem simulation of high-frequency electromagnetic fields, 46 (2010), pp. $2735-27384$.

[14] M. Grote and T. Mitkova, Explicit local time-stepping methods for Maxwell's equations, J. Comput. Appl. Math., 234 (2010), pp. 3283-3302.

[15] J. Hesthaven and T. Warburton, Nodal Discontinuous Galerkin Methods: Algorithms, Analysis, and Applications, vol. 54 of Texts in Applied Mathematics, Springer-Verlag, New York, 2008.

[16] J. S. Hesthaven And T. WARBURton, Nodal high-order methods on unstructured grids. I. Time-domain solution of Maxwell's equations, J. Comput. Phys., 181 (2002), pp. 186-221.

[17] M. HochBrUCK AND A. STURM, Error analysis of a second order locally implicit method for linear Maxwell's equations, CRC 1173-Preprint 2015/1, Karlsruhe Institute of Technology, 2015. https://na.math.kit.edu/marlis/research/publications.php.

[18] Y. Huang and J. Li, Interior penalty Discontinuous Galerkin method for Maxwell's equations in cold plasma, J. Sci. Comput., 41 (2009), pp. 321-340.

[19] Y. HuAng, J. LI, AND W. YANG, Interior penalty Discontinuous Galerkin methods for Maxwell's equations in dispersive media, J. Comput. Phys., 230 (2011), pp. 4559-4570.

[20] U. Inan and R. Marshall, Numerical Electromagnetics: The FDTD Method, Cambridge University Press, California, 2011.

[21] D. JIAO AND J.-M. JIN, Time-domain Finite-Element modeling of dispersive media, IEEE Microw. Wirel. Compon. Lett., 11 (2001), pp. 220-223.

[22] R. Joseph, S. Hagness, and A. Taflove, Direct time integration of Maxwell's equations in linear dispersive media wi th absorption for scattering and propagation of femtosecond electromagnetic pulses, Optics Lett., 16 (1991), pp. 1412-1414. 
[23] I. Karanasiou, K. Karathanasis, A. Garetos, and N. Uzunoglu, Development and laboratory testing of a noninvasive intracranial focused hyperthermia system, IEEE Trans. Microwave Theory Tech., 56 (2008), pp. 2160-2171.

[24] T. Kashiwa and I. Fukai, A treatment by fdtd method of dispersive characteristics associated with electronic polarization, Microwave Optics Tech. Lett., 3 (1990), pp. 203-205.

[25] D. Kelley and R. Luebbers, Piecewise linear recursive convolution for dispersive media using fdtd, IEEE Trans. Antennas Propagat., 44 (1996), pp. 792-797.

[26] M. Klemm, J. Leendertz, D. Gibbins, I. Craddock, A. Preece, and R. Benjamin, Towards contrast enhanced breast imaging using ultra-wideband microwave radar system, in IEEE Radio and Wireless Symposium. Special session on biomedical applications, New Orleans, USA, 2010, pp. 516-519.

[27] S. LANTERI AND C. SCheid, Convergence of a Discontinuous Galerkin scheme for the mixed time domain Maxwell's equations in dispersive media, IMA J. Numer. Anal., 33 (2013), pp. 432-459.

[28] J. LI, Unified analysis of leap-frog methods for solving time-domain Maxwell's equations in dispersive media, J. Sci. Comput., 47 (2011), pp. 1-26.

[29] T. Lu, W. CAI, AND P. Zhang, Discontinuous Galerkin Time domain methods for GPR simulation in dispersive media, IEEE Trans. Geosc. Rem. Sens., 43 (2005), pp. 72-80.

[30] T. Lu, P. Zhang, And W. CAI, Discontinuous Galerkin methods for dispersive and lossy Maxwell's equations and PML boundary conditions, IEEE Trans. Geosc. Rem. Sens., 200 (2004), pp. 549-580.

[31] R. Luebbers and F. Hunsberger, Fdtd for nth-order dispersive media, IEEE Trans. Antennas Propagat., 40 (1992), pp. 1297-1301.

[32] R. Luebbers, F. Hunsberger, K. Kunz, R. Standler, and M. Schneider, A frequencydependent Finite-difference Time-domain formulation formulation dispersive materials, IEEE Trans. on Electr. Comp., 32 (1990), pp. 222-227.

[33] D. MiklavČič, D. Šermov, H. Mekid, And L. Mir, A validated model of in vivo electric field distribution in tissues for electrochemotherapy and for DNA electrotransfer for gene therapy, Biochimica et Biophysica Acta, 1523 (2000), pp. 73-83.

[34] L. Moya, Temporal convergence of a locally implicit Discontinuous Galerkin method for Maxwell's equations, ESAIM: M2AN, 46 (2012), pp. 1225-1246.

[35] — Locally implicit Discontinuous Galerkin Time-domain methods for electromagnetic wave propagation in biological tissues, $\mathrm{PhD}$ thesis, Nice Sophia Antipolis University, 2013.

[36] D. SÁrmány, M. Botchev, and J. V. Der Vegt, Dispersion and dissipation error in highorder runge-kutta Discontinuous Galerkin discretisations of the Maxwell equations, J. Sci. Comput., 33 (2007), pp. 47-74.

[37] J. Schea, P. Kosmas, B. V. Veen, and S. Hagness, Contrast-enhanced microwave imaging of breast tumors: a computational study using $3 D$ realistic numerical phantoms, Inverse Problems, 26 (2010).

[38] B. Seny, J. Lambrechts, R. Comblen, V. Legat, and J.-F. Remacle, Multirate time stepping for accelerating explicit discontinuous galerkin computations with application to geophysical flows, 71 (2013), pp. 41-64.

[39] G. SERŠA, Application of electroporation in electrochemotherapy of tumors, in Electroporation based Technologies and Treatment. International scientific workshop and postgraduate course, Ljubljana, Slovenia, 2005, pp. 42-45.

[40] S. Sukharev, V. Klenchin, S. Serov, L. Chernomordik, and Y. Chizmadzhev, Electroporation and electrophoretic DNA transfer into cells: the effect of DNA interaction with electropores, Biophys J., 63 (1992), pp. 1320-1327.

[41] A. Taflove and C. Hagness, Computational Electrodynamics: The Finite-Difference TimeDomain Method, MA: Artech House, Norwood, third ed., 2005.

[42] T. Tsong, Electroporation of cell membranes, Biophys J., 60 (1991), pp. 297-306.

[43] J. Verwer, Component splitting for semi-discrete Maxwell equations, BIT Numer. Math., 51 (2010), pp. 427-445.

[44] D. Šel, D. Cukjati, D. Batiuskaite, T. Slivnik, L. Mir, and D. MiklavČič, Sequential Finite Element model of tissue electropermeabilization, IEEE Trans. Biomed. Engrg., 52 (2005), pp. 816-827.

[45] B. Wang, Z. XIE, AND Z. Zhang, Error analysis of a Discontinuous Galerkin method for Maxwell's equations in dispersive media, J. Comput. Phys., 229 (2010), pp. 8552-8563. 